\title{
Gaussian phase sensitivity of boson-sampling-inspired strategies
}

\author{
Antonio A. Valido* and Juan José García-Ripoll ${ }^{\circ}$ \\ Instituto de Física Fundamental IFF-CSIC, Calle Serrano 113b, 28006 Madrid, Spain
}

(Received 14 September 2020; accepted 8 March 2021; published 24 March 2021)

\begin{abstract}
In this paper we study the phase sensitivity of generic linear interferometric schemes using Gaussian resources and measurements. Our formalism is based on the Fisher information. This allows us to separate the contributions of the measurement scheme, the experimental imperfections, and auxiliary systems. We demonstrate the strength of this formalism using a broad class of multimode Gaussian states that includes well-known results from single- and two-mode metrology scenarios. Using this, we prove that input coherent states or squeezing improve upon the nonclassical states proposed in preceding boson-sampling-inspired phase-estimation schemes. We also develop a polychromatic interferometric protocol, demonstrating an enhanced sensitivity with respect to two-mode squeezed-vacuum states, for which the ideal homodyne detection is formally shown to be optimal.
\end{abstract}

DOI: 10.1103/PhysRevA.103.032613

\section{INTRODUCTION}

During the last decade a considerable attention has been devoted to figure out the optimal phase-estimation scheme for a (linear) photonic interferometer using Gaussian states and ideal quadrature measurements [1-5] by means of the celebrated parameter estimation theory [6-18]. In this context, most theoretical and experimental treatments have paid attention to the so-called quantum Fisher information (QFI), which dictates the ultimate phase sensitivity under generic measurements [7-9,19]. Interestingly, the optimal phase scheme able to attain the QFI could be determined via the symmetric logarithmic derivative (SLD) [2-5,20], though it displays an intricate dependence on the desired parameter, which represents a major obstacle at the experimental level. For instance, the QFI has been intensively studied for noisy and lossy two-mode Mach-Zehnder interferometers (MZIs) pumped by either a cross product of coherent and squeezed-vacuum states $[21,22]$ or a two-mode squeezed-vacuum state [22-25]. The phase sensitivity of the multimode scenario is less understood [3,5,26-32]. Recent work suggests that, in the case of decoherence-free Gaussian resources with fixed average number of photons, the optimal Heisenberg limit (HL) is reached with a trivial squeezed-vacuum state [29].

Most optimal Gaussian protocols relying on the QFI involve nontrivial technical challenges [33-35], such as engineering the passive transformation and generating highintensity [26] or highly squeezed light beams. In view of these problems, when working with experimental constraints we must focus on the Fisher information (FI) $[36,37]$ for the resources at hand-families of states, transformations, and measurements. This task has been completed in the singlemode MZI scenario [38], and in some cases also for the multimode setup $[18,20,39]$. More recently, the FI has permitted researchers to show the Heisenberg scaling in multimode

\footnotetext{
*a.valido@iff.csic.es
}

interferometric schemes endowed with single-mode squeezing resources and some preliminary classical knowledge about the parameter $[40,41]$. The FI approach is a versatile treatment to study the phase resolution of general circuits, such as reconfigurable photonic circuits $[33,35,42,43]$ with homodyne measurements. It is complementary to earlier and more difficult studies based on the quantum fidelity $[26,30,36,44]$.

In this paper we compute the Fisher information of arbitrary multimode interferometers working with Gaussian input states and Gaussian measurements (without necessarily assuming nonpassive resources and pre- or postprocessing treatments). This allows us to envisage strategies retrieving a reasonable compromise between the phase sensitivity and the technical constraints upon the experimental resources. For instance, we tackle the question whether multimode setups can provide a metrological advantage, or improve upon the shot-noise limit (SNL) with less demanding components than single-photon sources [45-48]. Our formalism also quantifies deviations from the ideal limits provided by the QFI and gives insight on the interplay between the experimental resources and imperfections, such as losses and nonideal detectors. On top of that, we analyze various interferometric schemes in terms of the resolution-energy tradeoff, and introduce a polychromatic protocol providing a multiplicative enhancement of the phase sensitivity with respect to the conventional strategy.

This paper is divided in two parts. In Sec. II we study the phase sensitivity of a linear, passive interferometer with Gaussian resources and measurements. In Sec. II A we introduce the phase-space formalism [1,49-51]. In Sec. II B we review the connection between phase estimation and the Fisher information. Section II B uses the phase-space formalism to compute the Fisher information of an arbitrary linear and passive interferometer with Gaussian input states and measurements. Our results connect the FI to the QFI, identifying contributions from the ancillas, the interferometer, and the measurement setup. The second part of this paper illustrates how the FI formalism can be applied to various setups. Section III A discusses an input state formed by 


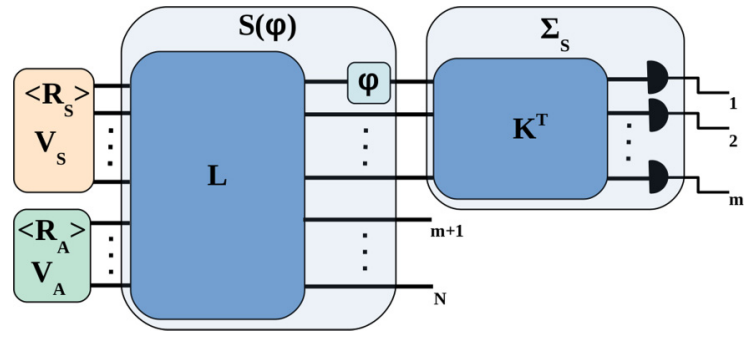

FIG. 1. Sketch of a generic $N$-mode Gaussian phase-estimation strategy consisting of a probe $m$-mode state, characterized by $\left\langle\boldsymbol{R}_{S}\right\rangle$ and $\boldsymbol{V}_{S}$ (orange), and an ancilla $(N-m)$-mode state, characterized by $\left\langle\boldsymbol{R}_{A}\right\rangle$ and $\boldsymbol{V}_{A}$ (green). Both probe and ancillary systems interact via the interferometer modeled by $\boldsymbol{L}$, whereafter the first probe mode undergoes the (single) phase rotation $\varphi$, such that the whole propagation is described by $S(\varphi)$. The output modes of the probe system are finally assessed by a generic quadrature measurement determined by $\Sigma_{S}$.

single-mode squeezed-vacuum and coherent states on $N-1$ auxiliary modes. Section III B introduces an interferometric scheme with polychromatic light. Finally, in Sec. IIIC we show how to introduce losses and nonideal detectors.

\section{GAUSSIAN PHASE SENSITIVITY}

Our paper is devoted to studying an interferometric setup [11,13-15] such as the one in Fig. 1. This phase-estimation scheme consists of (i) an $N$-mode input state of light $\hat{\rho}$ with $m$ principal models and $N-m$ auxiliary degrees of freedom that will be eventually discarded $[16,17,52,53]$, (ii) an interferometer $L$ that prepares the state of light prior to interaction, (iii) the actual phase transformation $\varphi$ that we wish to detect, and (iv) a final measurement stage that combines a linear transformation with local homodyne measurement on $m$ modes. This general scheme contains the MZI, and the vast majority of Gaussian (single) phase estimation previously treated as particular instances [21,22,26,29,38,54-56]. Our paper focuses on a family of Gaussian input states $[3,5]$ which we denote $\mathcal{G}\left(m, \bar{n}_{t}\right)$ and which is a product $\hat{\rho}=\hat{\rho}_{S} \otimes \hat{\rho}_{A}$, of a general Gaussian state $\hat{\rho}_{A}$ for the ancilla, and an isothermal state $\hat{\rho}_{S}$ for the system-i.e., an $m$-mode Gaussian state with a uniform number of thermal photons $\bar{n}_{t}$ on each mode. On the output of the interferometer, we consider a general $m$-mode homodyne detection scheme, engineered by an interferometer $K$ and local homodyne measurements. Finally, without loss of generality, we assume that the measured phase $\varphi$ acts as a local rotation $\hat{U}=\exp (-i \varphi \hat{H})$ on one of the modes.

We will now proceed in three steps. The following section will introduce the phase-space formalism, explaining how to express states $\hat{\rho}$, interferometers, local phase rotations, and measurements. Later in Sec. II B, we will introduce the Cramer-Rao bound and how the Fisher information determines the maximum achievable sensitivity of our interferometer. Finally, Sec. IIC connects both formalisms, providing an explicit formula for the Fisher information and the phase sensitivity of our setup, expressed in terms of the first and second moments of the input state, the covariance (CV) matrix of the measurement, and the passive transformations $L$ and $K$.

\section{A. Phase-space formalism}

We model the light using two quadratures per mode, $\hat{q}_{i}$ and $\hat{p}_{i}$, which satisfy the canonical commutation relations $\left[\hat{q}_{i}, \hat{p}_{j}\right]=i\left[\boldsymbol{J}_{N}\right]_{i j}$. Here we have introduced the symplectic form [49,51] $\boldsymbol{J}_{N}=\bigoplus_{i=1}^{N} \boldsymbol{J}$, expressed in terms of $[J]_{\alpha \beta}=\varepsilon_{\alpha \beta}$, the Levi-Civita symbol in two dimensions $\varepsilon_{\alpha \beta}$. Any operator $\hat{O}$ is described in terms of the Weyl symbol $W_{O}(\boldsymbol{R})$ spanned by the phase-space basis $\boldsymbol{R}=$ $\left(q_{1}, p_{1}, \ldots, q_{N}, p_{N}\right)^{T} \in \mathbb{R}^{2 N}$ with support in the real symplectic space $\left(\mathbb{R}^{2 N}, \boldsymbol{J}_{N}\right)[50,51]$. Gaussian states are those the density matrix of which has a Weyl symbol $W(\boldsymbol{V},\langle\boldsymbol{R}\rangle)$ that is fully determined by the first moments $\langle\boldsymbol{R}\rangle \in \mathbb{R}^{2 N}$ and the $\mathrm{CV}$ matrix:

$$
\boldsymbol{V}=\frac{1}{2}\left\langle\left\{\boldsymbol{R}, \boldsymbol{R}^{T}\right\}\right\rangle \in \mathbb{R}^{2 N \times 2 N} .
$$

In particular, our input state $\hat{\rho}$ is a tensor-product Gaussian state with first-moment vector $\langle\boldsymbol{R}\rangle=\left(\left\langle\boldsymbol{R}_{S}\right\rangle,\left\langle\boldsymbol{R}_{A}\right\rangle\right)^{T}$ and $\mathrm{CV}$ matrix $\boldsymbol{V}=\boldsymbol{V}_{S} \oplus \boldsymbol{V}_{A}$. Moreover, for our isothermal states $\boldsymbol{V}_{S}=\left(2 \bar{n}_{t}+1\right) \boldsymbol{S}^{\prime} \boldsymbol{I}_{m} \boldsymbol{S}^{\prime T}$, where $\boldsymbol{S}^{\prime}$ is an arbitrary $m$-mode (active or passive) symplectic transformation and $\boldsymbol{I}_{m}$ is the $2 m \times$ $2 m$ identity matrix. This set of states satisfies a symplecticlike identity $\boldsymbol{V}_{S} \boldsymbol{J}_{m} \boldsymbol{V}_{S}=\left(2 \bar{n}_{t}+1\right)^{2} \boldsymbol{J}_{m}$, implying a relation

$$
\boldsymbol{V}_{S}=\left(2 \bar{n}_{t}+1\right)^{2} \boldsymbol{J}_{m} \boldsymbol{V}_{S}^{-1} \boldsymbol{J}_{m}^{T}
$$

that will be extensively used throughout this paper.

The initial state undergoes a multimode interferometer transformation, given by a $2 N \times 2 N$ orthogonal, symplectic matrix [49]. For convenience, we split this matrix into system and ancilla:

$$
\boldsymbol{L}=\left(\begin{array}{cc}
\boldsymbol{L}_{S} & \boldsymbol{L}_{S A} \\
\boldsymbol{L}_{A S} & \boldsymbol{L}_{A}
\end{array}\right),
$$

where $\boldsymbol{L}_{S A}$ is a $2 m \times 2(N-m)$ isometry, while $\boldsymbol{L}_{S}$ is a nonorthogonal $2 m \times 2 m$ matrix which satisfies a symplecticlike relation $\boldsymbol{L}_{S}=\boldsymbol{J}_{m}^{T} \boldsymbol{L}_{S} \boldsymbol{J}_{m}$ as well.

After this preparation, the bosonic system suffers an unknown phase shift $\hat{U}(\varphi)$, generated by the operator

$$
\hat{H}=\frac{1}{4}\left(\hat{q}_{1}^{2}+\hat{p}_{1}^{2}\right)-\frac{1}{2} .
$$

The phase shift $\hat{U}$ induces a rotation in phase space $\boldsymbol{U}_{N}(\varphi)=$ $\boldsymbol{U}(\varphi) \oplus \boldsymbol{I}_{N-1}$, with $\boldsymbol{U}(\varphi)$ given by Eq. (A5).

The combined $N$-mode transformation $\boldsymbol{S}(\varphi)$ is composed of an $2 m \times 2 m$ nonorthogonal (nonsingular) matrix $\boldsymbol{S}_{S}$ acting solely upon the probe system, and an isometry $\boldsymbol{S}_{S A}(\varphi)$ describing the interference between the system and the ancillas:

$$
\begin{gathered}
\boldsymbol{S}_{S}(\varphi)=\boldsymbol{U}_{m}(\varphi) \boldsymbol{L}_{S}, \\
\boldsymbol{S}_{S A}(\varphi)=\boldsymbol{U}_{m}(\varphi) \boldsymbol{L}_{S A} .
\end{gathered}
$$

Since $\boldsymbol{S}(\varphi)$ describes a passive interferometric evolution, the following relations must hold [1]:

$$
\begin{gathered}
\boldsymbol{S}_{S}(\varphi) \boldsymbol{S}_{S}^{T}(\varphi)=\boldsymbol{I}_{m}-\boldsymbol{S}_{S A}(\varphi) \boldsymbol{S}_{S A}^{T}(\varphi), \\
\boldsymbol{S}_{S}(\varphi)=\boldsymbol{J}_{m}^{T} \boldsymbol{S}_{S}(\varphi) \boldsymbol{J}_{m}, \text { for } \varphi \in \mathbb{R},
\end{gathered}
$$

which also shall be used in the subsequent derivation.

The phase-estimation task is finally accomplished by performing a $m$-mode Gaussian measurement with outcome $\lambda \in$ $\mathbb{R}^{2 m}$. Any $m$-mode general-dyne measurement acting as a 
Gaussian positive operator-valued measure (POVM) $\hat{\Pi}_{\lambda}$ is characterized by a $2 m \times 2 m$ real, symmetric, and positivedefinite $\mathrm{CV}$ matrix:

$$
\boldsymbol{\Sigma}_{S}=\boldsymbol{K} \bigoplus_{j=1}^{m}\left(\begin{array}{cc}
r_{j} & 0 \\
0 & \frac{1}{r_{j}}
\end{array}\right) \boldsymbol{K}^{T} .
$$

$\boldsymbol{K}$ is an orthogonal symplectic transformation $[51,57,58]$ that may be implemented by the same or a different interferometer. The squeezing parameter $r_{j}=\left(1-\tau_{j}\right) / \tau_{j}$ is a function by the transmissivity $\tau_{j}$ of the measurement setup $[59,60]$. It includes the limit of an ideal homodyne measurement in the $q$ or $p$ quadratures as $r_{j} \rightarrow 0$ and $r_{j}^{-1} \rightarrow 0$, respectively. In the applications of Sec. III we will consider the ideal $N$ mode homodyne detection scheme consisting of identical local quadrature measurements, with $\boldsymbol{K}=\boldsymbol{I}_{N}$ and $r_{i}=r$.

We can compute the probability $p(\lambda \mid \varphi)$ of obtaining a measurement outcome $\lambda$ conditioned to a phase shift $\varphi$. This is a Gaussian function characterized by the first-moment vector and the CV matrix [57]:

$$
\begin{gathered}
\langle\lambda(\varphi)\rangle=\underbrace{\boldsymbol{S}_{S}(\varphi)\left\langle\boldsymbol{R}_{S}\right\rangle}_{\left\langle\lambda_{S}(\varphi)\right\rangle}+\boldsymbol{S}_{S A}(\varphi)\left\langle\boldsymbol{R}_{A}\right\rangle, \\
\boldsymbol{\sigma}(\varphi)=\underbrace{\boldsymbol{\Sigma}_{S}+\boldsymbol{S}_{S}(\varphi) \boldsymbol{V}_{S} \boldsymbol{S}_{S}^{T}(\varphi)}_{\boldsymbol{\sigma}_{S}(\varphi)}+\boldsymbol{S}_{S A}(\varphi) \boldsymbol{V}_{A} \boldsymbol{S}_{S A}^{T}(\varphi) .
\end{gathered}
$$

Note how the probe system statistics $\hat{\rho}_{S}$ only appears in $\left\langle\lambda_{S}(\varphi)\right\rangle$ and $\sigma_{S}$.

\section{B. Basics of phase-estimation theory}

Using the so-called maximum likelihood and Bayesian estimators [11,54,61], we can approximate an unknown phase shift $\varphi$ from a set of measurement outcomes $\lambda$. The precision of this method will be determined by the conditional probability $p(\lambda \mid \varphi)$, as well as the estimator strategy $p_{\text {est }}(\tilde{\varphi} \mid \lambda)$. The statistical inference process is described by the probability distribution [7,15,62]:

$$
P(\tilde{\varphi} \mid \varphi)=\int d^{2 m} \lambda p_{\text {est }}(\tilde{\varphi} \mid \lambda) p(\lambda \mid \varphi) .
$$

The quality of the estimator, or its precision, is given by the mean square error $[7,11,14]$ :

$$
\operatorname{var}(\varphi)=\left\langle\left\langle(\tilde{\varphi}-\varphi)^{2}\right\rangle\right\rangle=\int d \tilde{\varphi}(\tilde{\varphi}-\varphi)^{2} P(\tilde{\varphi} \mid \varphi) .
$$

In particular, for any unbiased estimator function with $\varphi=$ $\langle\langle\tilde{\varphi}\rangle\rangle$, the ultimate precision satisfies the Cramér-Rao bound $[7-9,14]$ :

$$
\operatorname{var}(\varphi) \geqslant \frac{1}{F(\varphi)},
$$

where $F(\varphi)$ is the Fisher information of the probability distribution $p(\lambda \mid \varphi)$ [8,9] [see Eq. (A1) in Appendix A]. In our Gaussian scenario, the FI can be explicitly computed (see Appendix A) as

$$
F(\varphi)=\partial_{\varphi}\left\langle\lambda^{T}\right\rangle \boldsymbol{\sigma}^{-1} \partial_{\varphi}\langle\lambda\rangle-\frac{1}{2} \operatorname{Tr}\left(\partial_{\varphi} \boldsymbol{\sigma}^{-1} \partial_{\varphi} \boldsymbol{\sigma}\right) .
$$

This includes earlier results for single- [26,38], two- [56], and multimode Gaussian metrology scenarios [5,15]. Notice that $F(\varphi)$ in the multiphase scenario is replaced by the Fisher information matrix [20,63], which could be expressed as Eq. (14) up to minor changes: $\partial_{\varphi}$ should be substituted by a gradient in the vector parameter, while $\sigma$ would be replaced by a larger matrix containing the parameter correlations due to the interferometric transformation. Since our framework relies on Eq. (14), it could be equivalently adapted to the multiphase situation as well by following the procedure illustrated in Sec. II C.

The Fisher information is particularized for a measurement strategy. The QFI is an upper bound over all POVM strategies, Gaussian or not $[7-9,14]$ :

$$
\mathcal{F}=\max _{\hat{\Pi}_{\lambda}}[F(\varphi)]
$$

Since by definition $F(\varphi) \leqslant \mathcal{F}$, it follows that the ultimate sensitivity $[19,62]$ for any quantum or classical measurement strategy is dictated by the quantum Cramér-Rao bound (QCRB) [7-9]:

$$
\operatorname{var}(\varphi) \geqslant \frac{1}{\mathcal{F}}
$$

As shown in Appendix A, there is a closed-form formula for the QFI when working with isothermal Gaussian input states and passive linear transformations $[2,26,44]$ :

$$
\begin{aligned}
\mathcal{F}= & \frac{1}{\left(2 \bar{n}_{t}+1\right)^{2}}\left[\left\langle\boldsymbol{R}_{1}^{\prime}\right\rangle^{T} \boldsymbol{V}_{1}^{\prime}\left\langle\boldsymbol{R}_{1}^{\prime}\right\rangle\right. \\
& \left.+\frac{1}{1+\left(2 \bar{n}_{t}+1\right)^{-2}}\left(\operatorname{Tr}\left(\boldsymbol{V}_{1}^{\prime} \boldsymbol{V}_{1}^{\prime}\right)-2\left(2 \bar{n}_{t}+1\right)^{2}\right)\right] .
\end{aligned}
$$

The first-moment $\left\langle\boldsymbol{R}_{1}^{\prime}\right\rangle$ and $\mathrm{CV} \boldsymbol{V}_{1}^{\prime}$ belong to the probe mode immediately before undergoing the phase-shift rotation. This expression is independent of $\varphi$ because of the phase-shift generator $\hat{H}(\varphi)=\hat{H}[9,62]$.

While $\mathcal{F}$ dictates the ultimate sensitivity limit, this limit requires implementing a measurement strategy that can depend on the estimator $\varphi$. This can involve elaborate transformations $\boldsymbol{L}$ and $\boldsymbol{K}$ and measurements of second- or higher-order moments of the quadrature. For this reason, unlike the vast majority of the previous works [7-9,11], we will center on discussing the FI and the attainable limits of phase sensitivity under given experimental setups and constraints. As we will show below, this is not a severe restriction. We can compute the sensitivity of protocols that are experimentally feasible (see Fig. 1). We can also show that it saturates the QCRB around certain strategies, and we can manipulate (14) to separate the contributions of the probe, the ancillary Gaussian state, the interferometer, and the Gaussian measurement scheme.

\section{FI analysis}

We now present the main result which is the basis of the future analysis. Starting from the identity (14), in Appendix B 
we decompose the FI as follows:

$$
\begin{aligned}
F(\varphi)= & \mathcal{F}_{S}+F_{\text {Anc }}(\varphi)+F_{\text {Int }}(\varphi)-F_{\text {Meas }}(\varphi) \\
& +\frac{\left(2 \bar{n}_{t}+1\right)^{2}}{1+\left(2 \bar{n}_{t}+1\right)^{2}}\left(\frac{\operatorname{Tr}\left(\boldsymbol{V}_{1}^{\prime} \boldsymbol{V}_{1}^{\prime}\right)}{\left(2 \bar{n}_{t}+1\right)^{4}}+2\right) \\
& -\operatorname{Tr}\left(\boldsymbol{P}_{0} \boldsymbol{L}_{S} \boldsymbol{L}_{S}^{T}\right),
\end{aligned}
$$

where $\mathcal{F}_{S}$ is the QFI associated to the $m$-mode probe system alone [which is obtained from (17) in the absence of the ancilla], and $\boldsymbol{P}_{\varphi}=\boldsymbol{U}(\varphi) \oplus \mathbf{0}_{m-1}$ is a $2 m \times 2 m$ projection matrix. The new functions $F_{\text {Anc }}(\varphi), F_{\text {Meas }}(\varphi)$, and $F_{\text {Int }}(\varphi)$, respectively, encode the influence of the input ancilla state, the $m$-mode quadrature measurement, and the interference between the ancilla and system. The measurement contribution reads

$$
\begin{aligned}
F_{\text {Meas }}(\varphi)= & \left\langle\boldsymbol{R}_{S}^{T}\right\rangle \partial_{\varphi} \boldsymbol{S}_{S}^{T} \tilde{\boldsymbol{\Sigma}}_{S} \partial_{\varphi} \boldsymbol{S}_{S}\left\langle\boldsymbol{R}_{S}\right\rangle \\
& -\frac{1}{2} \operatorname{Tr}\left(\partial_{\varphi} \tilde{\boldsymbol{\Sigma}}_{S} \partial_{\varphi}\left(\boldsymbol{S}_{S} \boldsymbol{V}_{S} \boldsymbol{S}_{S}^{T}\right)\right) .
\end{aligned}
$$

The symmetric and symplectic $2 m \times 2 m$ matrix

$$
\begin{aligned}
\tilde{\boldsymbol{\Sigma}}_{S}= & \left(\boldsymbol{S}_{S}^{T}\right)^{-1} \boldsymbol{V}_{S}^{-1} \boldsymbol{S}_{S}^{-1}\left(\boldsymbol{\Sigma}_{S}^{-1}+\left(\boldsymbol{S}_{S}^{T}\right)^{-1} \boldsymbol{V}_{S}^{-1} \boldsymbol{S}_{S}^{-1}\right)^{-1} \\
& \times\left(\boldsymbol{S}_{S}^{T}\right)^{-1} \boldsymbol{V}_{S}^{-1} \boldsymbol{S}_{S}^{-1}
\end{aligned}
$$

is manifestly independent of the input ancilla state. The influence of the ancilla is fully contained in

$$
\begin{aligned}
F_{\mathrm{Anc}}(\varphi)= & 2\left\langle\boldsymbol{R}_{S}^{T}\right\rangle \partial_{\varphi} \boldsymbol{S}_{S}^{T} \boldsymbol{\sigma}_{S}^{-1} \partial_{\varphi} \boldsymbol{S}_{S A}\left\langle\boldsymbol{R}_{A}\right\rangle \\
& +\left\langle\boldsymbol{R}_{A}^{T}\right\rangle \partial_{\varphi} \boldsymbol{S}_{S A}^{T} \boldsymbol{\sigma}_{S}^{-1} \partial_{\varphi} \boldsymbol{S}_{S A}\left\langle\boldsymbol{R}_{A}\right\rangle \\
& -\partial_{\varphi}\left\langle\lambda^{T}\right\rangle \tilde{\boldsymbol{V}}_{A} \partial_{\varphi}\langle\boldsymbol{\lambda}\rangle+\frac{1}{2} \operatorname{Tr}\left(\partial_{\varphi} \tilde{\boldsymbol{V}}_{A} \partial_{\varphi} \boldsymbol{\sigma}\right) \\
& -\frac{1}{2} \operatorname{Tr}\left(\partial_{\varphi} \boldsymbol{\sigma}_{S}^{-1} \partial_{\varphi}\left(\boldsymbol{S}_{S A} \boldsymbol{V}_{A} \boldsymbol{S}_{S A}^{T}\right)\right)
\end{aligned}
$$

with

$$
\tilde{\boldsymbol{V}}_{A}=\boldsymbol{\sigma}_{S}^{-1} \boldsymbol{S}_{S A}\left(\boldsymbol{V}_{A}^{-1}+\boldsymbol{S}_{S A}^{T} \boldsymbol{\sigma}_{S}^{-1} \boldsymbol{S}_{S A}\right)^{-1} \boldsymbol{S}_{S A}^{T} \boldsymbol{\sigma}_{S}^{-1} .
$$

Similarly, the function $F_{\text {Int }}(\varphi)$ only depends on the input system state and system-ancilla interference $S_{S A}$ [see Eq. (B9) in Appendix B]. Note that both $F_{\text {Anc }}(\varphi)$ and $F_{\text {Int }}(\varphi)$ vanish when the system-ancilla interference cancels (which corresponds to the nonassisted scenario without an ancilla system).

Let us give a brief overview about the derivation of the expression (18). From Eqs. (9) and (10) we may separate the contribution of the ancilla state. Indeed, using the so-called Woodbury identity [see Eq. (B1) in Appendix B] [64,65],

$$
F(\varphi)=F_{S}(\varphi)+F_{\text {Anc }}(\varphi),
$$

we can separate the contribution $F_{S}(\varphi)$ from the first moment $\left\langle\lambda_{S}(\varphi)\right\rangle$ and $\mathrm{CV} \sigma_{S}(\varphi)$. Collecting all remaining terms that depend on the auxiliary system, $F_{\text {Anc }}$ adopts the form in Eq. (21). This procedure may be repeated, using the symplecticlike identities (2) and (7), to separate from $F_{S}(\varphi)$ the interference $F_{\text {Int }}(\varphi)$ and measurement terms $F_{\text {Meas }}(\varphi)$, as shown in Eq. (B12). Finally, using property (B13), one may group the remaining terms into the QFI $\mathcal{F}_{S}$ [see Eq. (B14)] plus additional corrections, as shown in Eq. (18).

The closed-form expression (18) is valid for any probe isothermal Gaussian state $W\left(\boldsymbol{V}_{S},\left\langle\boldsymbol{R}_{S}\right\rangle\right) \in \mathcal{G}\left(m, \bar{n}_{t}\right)$, and for single-phase interferometric schemes, Gaussian ancilla states, as well as measurements. For the sake of clarity, we pay special attention to input coherent resources and a particular subset of quantum uniform multimode interferometers (QUMIs) recently studied in the context of boson-sampling inspired phase-estimation strategies [45-47]. These are further discussed in the following section.

\section{Coherent ancilla state and QUMI}

In the simple scenario in which the ancillary system is composed of coherent states $\boldsymbol{V}_{A}=\boldsymbol{I}_{N-m}$, that interfere with the system through a simple QUMI device-see the linear transformation $\boldsymbol{L}$ from Eq. (C2) - the FI simplifies to

$$
\begin{aligned}
F(\varphi)= & \tilde{F}_{S}(\varphi)+\left\langle\boldsymbol{R}_{A}^{T}\right\rangle \partial_{\varphi} \boldsymbol{S}_{S A}^{T} \boldsymbol{\sigma}^{-1} \partial_{\varphi} \boldsymbol{S}_{S A}\left\langle\boldsymbol{R}_{A}\right\rangle \\
& +2\left\langle\boldsymbol{R}_{S}^{T}\right\rangle \partial_{\varphi} \boldsymbol{S}_{S}^{T} \boldsymbol{\sigma}^{-1} \partial_{\varphi} \boldsymbol{S}_{S A}\left\langle\boldsymbol{R}_{A}\right\rangle
\end{aligned}
$$

with

$$
\boldsymbol{\sigma}=\underbrace{\left(\boldsymbol{\Sigma}_{S}+\boldsymbol{I}_{m}\right)}_{\tilde{\boldsymbol{\Sigma}}_{S}}+\boldsymbol{S}_{S} \underbrace{\left(\boldsymbol{V}_{S}-\boldsymbol{I}_{m}\right)}_{\tilde{\boldsymbol{V}}_{S}} \boldsymbol{S}_{S}^{T} .
$$

The term $\tilde{F}_{S}(\varphi)$ is the FI of a phase-estimation scheme that uses a Gaussian input state with first moment $\left\langle\boldsymbol{R}_{S}\right\rangle$ and CV $\tilde{\boldsymbol{V}}_{S}$, along with a Gaussian measurement with a white background noise $\tilde{\boldsymbol{\Sigma}}_{S}$

For a state with homogeneous input intensity $\bar{n}_{c}$ i.e., $\left\langle\boldsymbol{R}_{i}\right\rangle=\left(\sqrt{2 \bar{n}_{c}}, \sqrt{2 \bar{n}_{c}}\right)$ for $i \in[1, N]$-it turns out that $\boldsymbol{S}_{S A}\left\langle\boldsymbol{R}_{A}\right\rangle=(N-m) / m \boldsymbol{S}_{S}\left\langle\boldsymbol{R}_{S}\right\rangle$ [which follows from the transformation (C2)]. This means that the ancillary terms in Eq. (24) are positive and increase the FI-provided $\tilde{\boldsymbol{V}}_{S}$ is a positive semidefinite matrix. The auxiliary coherent state improves the phase sensitivity, although it introduces some background noise in the measurement outcome.

This result simplifies in the ideal homodyne detection in which the system, not only the ancilla, is in a coherent state $\boldsymbol{V}_{S}=\boldsymbol{I}_{m}$. Furthermore, the studied subset of QUMI schemes has the property that it maps a superposition of all input modes to the single mode that experiences the phase transformation [46]. In our notation, this mode has label 1 (see Fig. 1), so that

$$
\bar{n}_{1}=\frac{1}{4}\left(\left\langle\sum_{i=1}^{N} \frac{1}{\sqrt{N}} \boldsymbol{R}_{i}\right\rangle\right)^{2}=\frac{N}{2}\left(\sqrt{2 \bar{n}_{c}}\right)^{2}=N \bar{n}_{c} .
$$

The ancilla proves beneficial as increases phase sensitivity, since $\tilde{F}_{S}(\varphi)$ becomes $4 m \bar{n}_{c}$ in the optimal operating points $\varphi_{\mathrm{opt}}=\mp \pi / 4$ [see the discussion around Eq. (36) in Sec. III A]. Using Eq. (24) we obtain the phase sensitivity for the QUMI assisted coherent setup:

$$
(\delta \varphi)^{2}=\frac{1}{4 \bar{n}_{c} N},
$$

in agreement with previous results for single-parameter schemes with an external phase reference [16,17]. This coincides with the phase sensitivity of a single-mode coherent state with input intensity $\bar{n}_{c} N$. Moreover, Eq. (27) shows that input coherent resources outperform earlier QUMI-based phase estimation using single-photon states [45-48], for any size of the interferometer. For more general assisted phaseestimation schemes, it is less clear to see the influence owing to the interferometer $F_{\text {Int }}(\varphi)$ and ancilla $F_{\text {Anc }}(\varphi)$ contributions at first sight; instead they deserve a more profound 
analysis that is beyond the scope of the present treatment $[16,17,52,53]$.

\section{APPLICATION: $N$-MODE HOMODYNE DETECTION WITHOUT AN ANCILLA SYSTEM}

We will now compare the strength of our treatment with earlier Gaussian phase-estimation analysis [21,22,26,29,38], using no auxiliary modes $(N=m)$, Gaussian pure input states $\left(\bar{n}_{t}=0\right)$, and an ideal $N$-mode homodyne measurement. Since there are no ancillas, we can eliminate the subscript $S$, $\boldsymbol{S}_{S}(\varphi) \rightarrow \boldsymbol{S}(\varphi),\left\langle\boldsymbol{R}_{S}^{T}\right\rangle \rightarrow\left\langle\boldsymbol{R}^{T}\right\rangle, \boldsymbol{V}_{S} \rightarrow \boldsymbol{V}$, and $\mathcal{F}_{S} \rightarrow \mathcal{F}$. Both the FI (18)

$$
F(\varphi)=\mathcal{F}-F_{\text {Meas }}(\varphi)+\frac{1}{2}\left[\operatorname{Tr}\left(\boldsymbol{V}_{1}^{\prime} \boldsymbol{V}_{1}^{\prime}\right)-2\right]
$$

and the contribution from the measurement radically simplify [see Eq. (B15) in Appendix B]:

$$
\begin{aligned}
F_{\text {Meas }}(\varphi)= & \left\langle\boldsymbol{R}^{T}\right\rangle \boldsymbol{L}^{T} \boldsymbol{P}_{\varphi}^{T} \boldsymbol{S} \boldsymbol{V} \boldsymbol{S}^{T} \tilde{\boldsymbol{\Sigma}} \boldsymbol{S} \boldsymbol{V} \boldsymbol{S}^{T} \boldsymbol{P}_{\varphi} \boldsymbol{L}\langle\boldsymbol{R}\rangle \\
& -\frac{1}{2} \operatorname{Tr}\left(\left(\tilde{\boldsymbol{\Sigma}} \partial_{\varphi}\left(\boldsymbol{S} \boldsymbol{V} \boldsymbol{S}^{T}\right)\right)^{2}\right) \\
& +\operatorname{Tr}\left(\tilde{\boldsymbol{\Sigma}} \boldsymbol{S} \boldsymbol{V} \boldsymbol{S}^{T}\left(\partial_{\varphi}\left(\boldsymbol{J}_{N} \boldsymbol{S} \boldsymbol{V} \boldsymbol{S}^{T}\right)\right)^{2}\right) .
\end{aligned}
$$

The matrix $\Sigma$ that characterizes the Gaussian measurement appears in the new matrix $\tilde{\boldsymbol{\Sigma}}=\left(\boldsymbol{\Sigma}+\boldsymbol{S} \boldsymbol{V} \boldsymbol{S}^{T}\right)^{-1}$. For an ideal homodyne detection in either position or momentum quadrature, $\boldsymbol{\Sigma}$ effectively becomes a projection matrix, $\boldsymbol{\pi}^{(x)}=$ $\operatorname{diag}(1,0,1,0, \cdots, 1,0)$ or $\boldsymbol{\pi}^{(p)}=\operatorname{diag}(0,1,0,1, \cdots, 0,1)$, respectively. In this case $\tilde{\boldsymbol{\Sigma}}$ must be understood as a MoorePenrose (MP) inverse [51,58,66], computed as follows [64]:

$$
\tilde{\boldsymbol{\Sigma}}^{(x / p)}=\left(\boldsymbol{\pi}^{(x / p)} \boldsymbol{S} \boldsymbol{V} \boldsymbol{S}^{T} \boldsymbol{\pi}^{(x / p)}\right)^{\mathrm{MP}} .
$$

Note also that the CV matrix of the chosen measurement remains invariant $\boldsymbol{\Sigma}=\boldsymbol{K} \boldsymbol{\Sigma} \boldsymbol{K}^{T}$ under any interferometric transformation $\boldsymbol{K}$, rendering this choice irrelevant [67].

\section{A. Coherent and one-mode squeezed resources}

Let us analyze a collection of independent single-mode squeezed states, characterized by an arbitrary displacement $\langle\boldsymbol{R}\rangle \in \mathbb{R}^{2 N}$ and the CV matrix:

$$
\boldsymbol{V}=\boldsymbol{V}_{1}\left(s_{1}\right) \bigoplus \boldsymbol{V}_{N-1}\left(s_{2}\right)
$$

with

$$
\boldsymbol{V}_{l}(s)=\bigoplus_{i=1}^{l}\left(\begin{array}{ll}
s & 0 \\
0 & \frac{1}{s}
\end{array}\right) .
$$

The squeezing of the first and of the remaining $N-1$ modes is given by the parameters $s_{1}, s_{2} \in \mathbb{R}^{+}$. When $N=2$, this state reduces to the vast majority of nonentangled Gaussian states previously studied: when $s_{1}=s_{2}=s$, it maps to studies of single-mode squeezed states [3,21,38,54,56,68-72], when $s_{2}=1$ we have the squeezed mode combined with a coherent state from $[16,17,22,26,29,56,61,72,73]$, and for $s_{1}=s_{2}=1$ we recover the coherent phase-estimation scenario and the SNL scaling.

The QFI depends of the average number of photons on the mode that undergoes the phase rotation (concretely, the QFI for pure probe states is proportional to the variance of the photon number $[9,46,54])$. We can therefore concentrate on the previously introduced QUMI setup, which maximizes this intensity. For this we find

$$
\boldsymbol{S}_{\mathrm{QUMI}} \boldsymbol{V} \boldsymbol{S}_{\mathrm{QUMI}}^{T}=\left(\begin{array}{cc}
\boldsymbol{\Omega}_{N}\left(\varphi, s_{1}, s_{2}\right) & \mathbf{0} \\
\mathbf{0} & \boldsymbol{V}_{N-2}\left(s_{2}\right)
\end{array}\right),
$$

where $\boldsymbol{\Omega}_{N}$ is a $4 \times 4$ real, symmetric matrix the representation of which does not affect the discussion [see Appendix $\mathrm{C}$ and Eq. (C3)]. Note how the size of (32) grows as $2\left(N_{s_{1}}+1\right) \times$ $2\left(N_{s_{1}}+1\right)$ for a large number $N_{s_{1}}$ of states with squeezing $s_{1}$. Replacing (32) in (30), we further obtain

$$
\tilde{\boldsymbol{\Sigma}}^{(x / p)}=\boldsymbol{A}^{(x / p)} \oplus \operatorname{diag}(1,0, \cdots, 1,0) .
$$

Here, $\boldsymbol{A}^{(x / p)}$ is a $4 \times 4$ real matrix given by Eqs. (C11) and (C12). By paying attention to (32), it is clear to see that the matrices within the trace in the expression (29) effectively play the role of a projection operator in the phase space supporting the mode undergoing the rotation, i.e., $\partial_{\varphi}\left(\boldsymbol{S}_{\mathrm{QUMI}} \boldsymbol{V} \boldsymbol{S}_{\mathrm{QUMI}}^{T}\right)=\partial_{\varphi} \boldsymbol{\Omega}_{N} \oplus \mathbf{0}_{N-1}$. Having evaluated the quantities (32) and (33), after substitution in (29) one obtains the FI:

$$
\begin{aligned}
F^{(x / p)}(\varphi)= & \mathcal{F}-\frac{1}{2 N^{2}}\left(a_{N}^{2}\left(s_{2}, s_{1}\right)+\frac{a_{N}^{2}\left(s_{1}, s_{2}\right)}{\left(s_{1} s_{2}\right)^{2}}-2 N^{2}\right) \\
& +f_{N}^{(x / p)}\left(\sin ^{2} \varphi, s_{1}, s_{2}\right) \\
& -\left\langle\boldsymbol{R}_{1}^{\prime}\right\rangle \boldsymbol{W}_{N}^{(x / p)}\left(\varphi, s_{1}, s_{2}\right)\left\langle\boldsymbol{R}_{1}^{\prime}\right\rangle
\end{aligned}
$$

where we have introduced $a_{N}\left(s_{1}, s_{2}\right)=(N-1) s_{1}+s_{2}$, two auxiliary functions $f_{N}^{(x)}, f_{N}^{(p)}$ [see Eqs. (C19) and (C20)], and a real symmetric matrix $\boldsymbol{W}_{N}^{(x / p)} \in \mathbb{R}^{2 \times 2}$ [see Eqs. (C13)-(C15)].

The optimal phase-estimation strategy for a given $\varphi$ must saturate the QCRB (16). In that case the last three terms in Eq. (34) cancel each other and $F=\mathcal{F}$. To illustrate, let us consider an input beam with the same coherent low intensity $\bar{n}_{c} \ll N$ in each mode [i.e., $\left.\left\langle\boldsymbol{R}_{i}\right\rangle=\left(\sqrt{2 \bar{n}_{c}}, \sqrt{2 \bar{n}_{c}}\right)\right]$. For this choice, the QFI takes the form

$$
\begin{aligned}
\mathcal{F}= & 2 \bar{n}_{c} N \frac{1+s_{2}^{2}}{s_{2}}+\frac{1}{2}\left(4 \bar{n}_{c}\left(s_{1}+\frac{1}{s_{1}}-s_{2}-\frac{1}{s_{2}}\right)\right. \\
& \left.+s_{2}^{2}+\frac{1}{s_{2}^{2}}-2\right)+O\left(N^{-1}\right) .
\end{aligned}
$$

The first term, proportional to $N$, reproduces the QFI of a coherent input state, and the second and third term cancel precisely for that type of input $s_{1}=s_{2}=1$. Moreover, we may expand Eq. (34) in the limit of large interferometers $1 \ll N$ with finite energy $1 / N \ll s_{1 / 2} \ll N$ :

$$
\begin{aligned}
& F^{(x / p)}(\varphi) \\
& =\frac{4 \bar{n}_{c} N s_{2}(1 \mp \sin (2 \varphi))}{1+s_{2}^{2} \mp\left(1-s_{2}\right) \cos (2 \varphi)} \\
& \quad+\frac{2 s_{1}^{-1}\left(s_{1}(1 \mp 1)+s_{2}(1 \pm 1)\right)}{\left(1+s_{2}^{2} \mp\left(1-s_{2}^{2}\right) \cos (2 \varphi)\right)^{2}}\left(\frac{s_{1}\left(1-s_{2}^{2}\right)^{2} \sin ^{2}(2 \varphi)}{s_{1}(1 \mp 1)+s_{2}(1 \pm 1)}\right. \\
& \left.\quad+\bar{n}_{c}\left(s_{1}-s_{2}\right)(1 \mp \sin (2 \varphi))\left(1-s_{2}^{2} \mp\left(1+s_{2}^{2}\right) \cos (2 \varphi)\right)\right) \\
& \quad+O\left(N^{-1}\right) .
\end{aligned}
$$



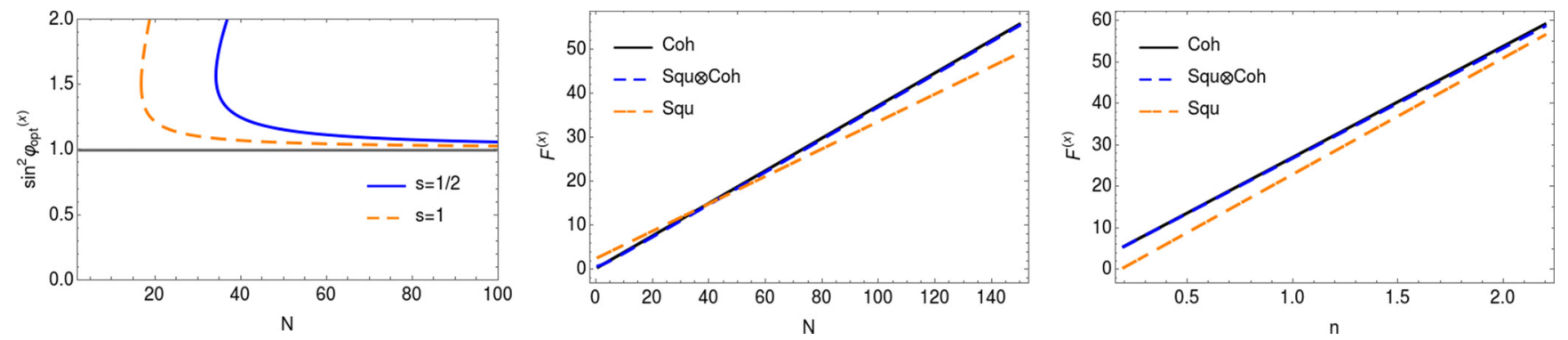

FIG. 2. Left: The roots of the polynomial (38) as functions of the interferometer size $N$, where the horizontal black line represents the unit value $\sin ^{2} \varphi_{0}^{(x)}=1$. Notice that the solid blue and dashed orange lines do not regard injective functions because the polynomial has two distinct real roots. Central: The FI associated to the position quadrature measurement as a function of the interferometer size and for distinct input probe states: the black-solid, blue-dashed, and orange-dot-dashed lines correspond to the tensor product of coherent (i.e., $s_{1}=s_{2}=1$ ), one-mode squeezed $\otimes$ coherent (i.e., $s_{1}=e^{2 s^{\prime}}$ and $s_{2}=1$ ), and single-mode squeezed (i.e., $s_{1}=s_{2}=e^{2 s^{\prime}}$ ) states, respectively. For a fair comparison, we have fixed the input mean photon number per mode to an identical value for all input states, i.e., $\bar{n} \approx 1.38$, and we have chosen the unknown phase shift $\varphi=\pi / 3$ and the squeezing parameter $s^{\prime}=1 / 2$. Right: Similarly, the FI as a function of the mean photon number per mode for a fixed squeezing parameter. We have taken the same values for the rest of the parameters.

Here the signs $\mp$ correspond to the use of position and momentum quadratures, respectively.

Inspecting Eq. (36) reveals that the leading sensitivity in $F^{(x / p)}$ resembles the QFI of coherent states (35) around the optimal working points $\varphi_{\mathrm{opt}}^{(x / p)}=\mp \pi / 4$, i.e.,

$$
F^{(x / p)}(\mp \pi / 4)=4 \bar{n}_{c} N+O\left(\left(s_{1}-1\right) N^{-1},\left(s_{2}-1\right) N^{-1}\right) .
$$

In other words, the combination of ideal homodyne detection and squeezed input resources with $s_{1} \neq s_{2}$ can approach the QCRB for large interferometers, though it never saturates the QFI except in the strict coherent limit $\left(s_{1} \rightarrow 1, s_{2} \rightarrow 1\right)$ in agreement with previous results for two-mode [21,22] and multimode interferometric schemes [26]. On the other hand, if we use displaced single-mode squeezed states $s_{1}=s_{2}=s$, the ideal homodyne detection is never an optimal measurement scheme: the three last terms in the right-hand side of Eq. (34) never cancel each other if $0<|\langle\boldsymbol{R}\rangle|$ and $0<s$.

For input resources with vanishing displacement, the optimal working point $\varphi_{\mathrm{opt}}^{(x / p)}$ is found by solving second-order equations in the variable $y=\sin ^{2} \varphi \rightarrow$ [see Eqs. (C19) and (C20)]. For instance, the condition to saturate the QCRB for a position quadrature measurement is

$$
y^{2} \alpha_{N}^{(x)}\left(s_{1}, s_{2}\right)+y \beta_{N}^{(x)}\left(s_{1}, s_{2}\right)+\delta_{N}^{(x)}\left(s_{1}, s_{2}\right)=0,
$$

with coefficients $\alpha_{N}^{(x)}, \beta_{N}^{(x)}$, and $\delta_{N}^{(x)}$ given by Eqs. (C21)(C23). In the particular situation of a homogeneous squeezing $s_{1}=s_{2}=e^{-2 s^{\prime}}$ with $s^{\prime} \in \mathbb{R}$, the QCRB is saturated for

$$
\cos \left(2 \varphi_{\mathrm{opt}}^{(x / p)}\right)= \pm \tanh \left(2 s^{\prime}\right)
$$

and Eq. (34) returns

$$
F^{(x / p)}\left(\varphi_{\mathrm{opt}}^{(x / p)}\right)=8 \bar{n}_{s^{\prime}}\left(\bar{n}_{s^{\prime}}+1\right),
$$

with $\bar{n}_{s^{\prime}}$ denoting the input average photon number per mode (i.e., $\bar{n}_{s^{\prime}}=\sinh ^{2} s^{\prime}$ ). Notice that this result holds for any choice of the interferometric transformation [66]. This coincides with the single-mode Gaussian state results, found with alternative methods based on the fidelity $[3,54,55,68]$ or the SLD [38].
The subsidiary condition (38) proves that a quadrature detection in position (or equivalently, in momentum) is no longer optimal for a tensor product of zero-displacement states with $s_{1}=s$ and $s_{2}=1$. We see this in the left panel of Fig. 2, which shows the real roots of (38) as a function of $N$ for two fixed squeezing values $s$. Note how these roots are always above or at most equal to 1 for all problem sizes $N$. Consequently, there is no value $\varphi_{\mathrm{opt}}^{(x)}$ for which the QCRB is saturated except for the single-mode Gaussian metrology setup $N=1$. This observation is also confirmed by computing the roots in the limits of extreme squeezing in either position or momentum, i.e., $\lim _{s \rightarrow \infty} \sin ^{2} \varphi_{\text {opt }}^{(x)}=N^{2} /(2 N-1)$. All these findings are consistent with results obtained in the single- and two-mode phase-estimation analysis based on the SLD [21,26,30,38,55]: for displaced squeezed states the SLD is a quadratic operator in terms of the quadrature operators $[1,2,5]$ (which means that the optimal measurement scheme is non-Gaussian), however it becomes linear when dealing with either coherent or squeezed-vacuum resources [38].

The FI is also plotted in Fig. 2 for the purpose of comparison. The central panel depicts this in terms of the interferometer size $N$ for a given homogeneous intensity $\bar{n}$, while the right panel illustrates it as a function of $\bar{n}$ at a fixed interferometer size $N=100$. In summary, these figures outline the main conclusion from Eq. (36): that is, none of the nonentangled Gaussian states along with the QUMI architecture provide a better scaling than the SNL (see the black solid line) in the finite energetic regime and for large interferometer sizes. In other words, our analysis indicates that QUMI-based phase-estimation strategies provide no real advantage with respect to the resolution-energy tradeoff [45-48].

\section{B. Two-mode squeezed resources and polychromatic phase generator}

Let us now study a metrology setup using two-mode nondegenerate squeezed states as resources. These states have been shown to overcome the SNL in estimation errors or phase sensitivities when using homodyne [24,74], intensity [75], or parity measurements [76-78]. The input state will 

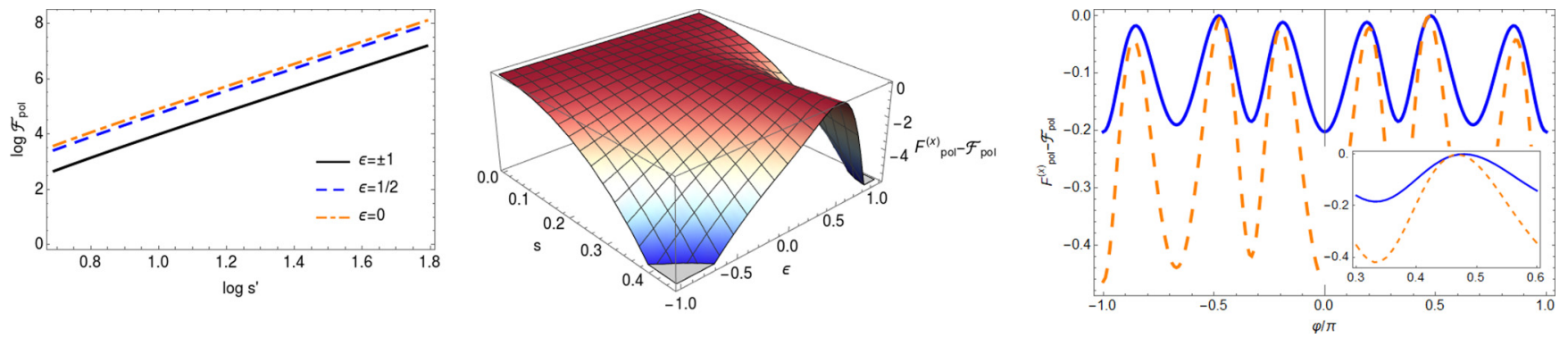

FIG. 3. Left: Log plot of the polychromatic QFI as a function of $s^{\prime}$ and for distinct values of the modulation parameter $\epsilon$, shown in the large squeezing regime. We have fixed the transmissivity $\tau=0$. Central: Three-dimensional plot of the deviation associated to the position quadrature measurement for input two-mode squeezed-vacuum states and fixed value of the phase shift $\varphi=\pi / 4$. Right: Similarly, the deviation of the FI as a function of $\varphi$ for a fixed modulation frequency $\epsilon=1 / 2$ and two given values of the squeezing parameter: the blue and dashed orange lines correspond to $s=0.1$ and 0.15 , respectively. In the central and left panels, the transmissivity was chosen $\tau=1 / 2$. Log denotes the natural logarithm.

be described by the first-moment vector $\langle\boldsymbol{R}\rangle \in \mathbb{R}^{4}$ and a CV matrix $[14,23,79]$ :

$$
\boldsymbol{V}=\left(\begin{array}{cccc}
\cosh 2 s^{\prime} & 0 & \sinh 2 s^{\prime} & 0 \\
0 & \cosh 2 s^{\prime} & 0 & -\sinh 2 s^{\prime} \\
\sinh 2 s^{\prime} & 0 & \cosh 2 s^{\prime} & 0 \\
0 & -\sinh 2 s^{\prime} & 0 & \cosh 2 s^{\prime}
\end{array}\right),
$$

that depends on the squeezing parameter $s^{\prime} \in \mathbb{R}^{+}$. Notice that one obtains the result related to the coherent resources discussed previously for the choice $s^{\prime}=0$.

These states can be generated using the well-established procedure of pumping a nondegenerate optimal parametric amplifier with a strong coherent beam, say at frequency $2 \omega_{0}$. These input photons are split into highly correlated pairs that conserve the total energy $\omega_{1,2}=\omega_{0} \pm \Omega$. Here $\Omega<\omega_{0}$ is a small modulation frequency that renders the photons distinguishable [50,76,77]. To the best of our knowledge, there is no previous treatment that studied the influence of such modulation from the metrological point of view (for instance, see $[28,69,80])$.

We will now go beyond previous phase-estimation analysis, addressing a polychromatic metrology scenario in which each port of the two-mode interferometric setup is fed with beams at two different frequencies. We label those modes with the annihilation operators $\hat{a}_{\omega_{1}}$ and $\hat{a}_{\omega_{2}}$, and consider that different frequencies may experience a different single-mode phase shift, generated by

$$
\hat{H}_{\mathrm{pol}}(\epsilon)=(1+\epsilon) \hat{n}_{\omega_{1}}+(1-\epsilon) \hat{n}_{\omega_{2}} .
$$

The parameter $-1 \leqslant \epsilon \leqslant 1$ can be regarded as a frequencydependent index of refraction or optical path, and $\hat{n}_{\omega_{i}}=$ $\hat{a}_{\omega_{i}}^{\dagger} \hat{a}_{\omega_{i}}$. As the total average energy $\left\langle\hat{H}_{\mathrm{pol}}(\epsilon)\right\rangle$ remains constant for distinct $\epsilon$, we can compare the resolution-energy tradeoff retrieved by polychromatic Gaussian phase-estimation scenarios. The choice (42) returns an extension of the phase-shift generator that is

$$
\boldsymbol{U}_{\mathrm{pol}}(\varphi, \epsilon)=\boldsymbol{U}((1+\epsilon) \varphi) \oplus \boldsymbol{U}((1-\epsilon) \varphi),
$$

which reduces to the conventional generator (A5) for the choices $\epsilon= \pm 1$ [74]. Further, we shall consider that the transformation $\boldsymbol{L}$ represents a beam splitter with transmissivity $\tau$.
Returning to the phase-space formalism, the polychromatic QFI can be expressed as follows:

$$
\begin{aligned}
\mathcal{F}_{\text {pol }}(\epsilon)= & (1+\epsilon)^{2} \mathcal{F}_{1}+(1-\epsilon)^{2} \mathcal{F}_{2} \\
& +4\left(1-\epsilon^{2}\right)\left[\operatorname{Tr}\left(\boldsymbol{V}_{12}^{\prime} \boldsymbol{V}_{12}^{\prime}\right)+2\left\langle\boldsymbol{R}_{1}^{\prime T}\right\rangle \boldsymbol{V}_{12}^{\prime}\left\langle\boldsymbol{R}_{2}^{\prime}\right\rangle\right],
\end{aligned}
$$

where $\quad \mathcal{F}_{1}=\mathcal{F}_{2}=(1+4 \tau(1-\tau)) \bar{n}_{s^{\prime}}\left(\bar{n}_{s^{\prime}}+2\right) \quad$ with $\quad \bar{n}_{s^{\prime}}$ denoting the total average number of photons, whereas $\left\langle\boldsymbol{R}_{i}^{\prime}\right\rangle=(\boldsymbol{L}\langle\boldsymbol{R}\rangle)_{i} \quad$ and $\quad \boldsymbol{V}_{12}^{\prime}=\operatorname{diag}\left((1-2 \tau) \sinh 2 s^{\prime},-(1-\right.$ $\left.2 \tau) \sinh 2 s^{\prime}\right)$.

The left panel of Fig. 3 shows a log-log plot of the QFI in terms of the squeezing parameter, for distinct choices of the frequency modulation and vanishing input displacement. The polychromatic QFI is larger than the monochromatic counterpart for sufficient high squeezing $\left(1 \ll s^{\prime}\right)$, and the highest sensitivity is obtained for $\epsilon=0$. Interestingly, the sensitivity grows with the squeezing with an identical power for all values of $\epsilon$, so that the polychromatic QFI may be approximately expressed as $\mathcal{F}_{\text {pol }}(\epsilon) \approx c(\epsilon, \tau) \mathcal{F}_{1}$ with $c$ being a multiplicative enhancement independent of $s^{\prime}$. This factor is found to take values $2 \lesssim c \lesssim 10$ for the available modulation frequencies and transmissivity, implying that a polychromatic setup can provide a significant improvement of the resolutionenergy tradeoff compared to the monochromatic MZI, e.g., $\mathcal{F}_{\text {pol }} \sim 10 \bar{n}_{s^{\prime}}^{2}$ for $1 \ll s^{\prime}, \epsilon=0$, and $\tau=0$.

The treatment about the FI presented in Sec. II C holds for very general phase generators beyond (4) and can be adapted to the polychromatic scenario. Going back to the general expression (B12) and replacing the phase-shift generator (43), we obtain a closed-form expression of the FI associated to the polychromatic strategy by following a similar procedure as to compute the expression (28) discussed in Sec. II C. The result is

$$
\begin{aligned}
F_{\mathrm{pol}}(\varphi, \epsilon)= & \mathcal{F}_{\mathrm{pol}}(\epsilon)-F_{\text {Meas }}(\varphi, \epsilon)-2\left(1+\epsilon^{2}\right) \\
& +\frac{1}{2} \operatorname{Tr}\left((1+\epsilon)^{2} \boldsymbol{V}_{1}^{\prime} \boldsymbol{V}_{1}^{\prime}+(1-\epsilon)^{2} \boldsymbol{V}_{2}^{\prime} \boldsymbol{V}_{2}^{\prime}\right) \\
& -2\left(1-\epsilon^{2}\right)\left(\operatorname{Tr}\left(\boldsymbol{V}_{12}^{\prime} \boldsymbol{V}_{12}^{\prime}\right)+3\left\langle\boldsymbol{R}_{1}^{\prime T}\right| \boldsymbol{V}_{12}^{\prime}\left\langle\boldsymbol{R}_{2}^{\prime}\right\rangle\right),
\end{aligned}
$$

where $F_{\text {Meas }}(\varphi, \epsilon)$ is obtained from (29) after substituting the CV matrix (41). 
The central panel of Fig. 3 displays the deviation of the Fisher information from the quantum limit $F_{\text {pol }}^{(x)}(\varphi, \epsilon)-$ $\mathcal{F}_{\text {pol }}(\epsilon)$, in the case of position quadrature measurements, for a fixed unknown phase shift and vanishing input displacement. As expected, the deviation is always negative or zero. However, it also remains close to zero for a growing squeezing around $\epsilon \approx \pm 1 / 2$. This indicates that an ideal $N$-mode quadrature detection may constitute an optimal measurement scheme. We can verify this for two-mode squeezed-vacuum states and $\epsilon= \pm 1$. After a 50:50 beam splitter transformation (i.e., $\tau=1 / 2$ ), the probe system is in the tensor product of single-mode squeezed-vacuum states. In agreement with the discussion in the previous section, we may expect to recover an identical relation for the operating point as Eq. (39). Indeed, after some manipulation Eq. (45) boils down to a simple algebraic expression in the argument $y=$ $\cos (4 \phi)$ [see Eqs. (C24) and (C25)], from which follows the subsidiary condition: $\cos \left(4 \phi_{\mathrm{opt}}^{(x / p)}\right)=\mp \epsilon /|\epsilon| \tanh \left(2 s^{\prime}\right)$. This is complementary to earlier findings for homodyne or intensity detection schemes combined with active interferometry $[25,56,75]$.

The right panel in Fig. 3 also illustrates the saturation of the QCRB, as zeros of the difference $F_{\text {pol }}^{(x)}(\varphi, \epsilon)-\mathcal{F}_{\text {pol }}(\epsilon)$, for a strategy based on position measurements and vanishing input displacement. Note how this deviation is an oscillating function of the phase, with an amplitude that grows with the squeezing [see dashed vs solid lines in Fig. 3]. Upon a closer look we appreciate an optimal operating point around $\varphi_{\mathrm{opt}}^{(x)} \approx \pi / 2$ (see Fig. 3 inset), where the FI reaches the QFI, and thus it takes the value

$$
F_{\mathrm{pol}}\left(\varphi_{\mathrm{opt}}^{(x)}, 1 / 2\right)=5 \sinh ^{2}\left(2 s^{\prime}\right) \approx 5 \bar{n}_{s^{\prime}}^{2} .
$$

This is an optimal measurement strategy for the polychromatic scenario (with $\tau=1 / 2$ ), a result which is also recovered in a setup with momentum-based measurements.

Unfortunately, the quadrature measurement is no longer optimal in the case of a vanishing modulation frequency $\epsilon=$ 0 , which is when the polychromatic scheme obtains the largest improvement over the conventional strategy. In this case, the optimal operating point is determined by an algebraic equation $f_{0}^{(x)}\left(\cos (4 \varphi), s^{\prime}\right)=0$ [see discussion around Eq. (C26) in Appendix C], as in preceding sections. The closed-form expressions for these roots given in Eq. (C29) show that no value of squeezing $0<s^{\prime}$ can saturate the QCRB for a given phase shift $\varphi$.

\section{Photon-loss effects and nonunit efficiency detection}

Finally, we address the degrading effects owing to the experimental imperfections, extending our treatment to include these in the analysis of the FI. In most interesting cases, the photon-loss process, determined by a given strength $\eta_{\text {loss }}$, and the nonunit efficiency detection, designated by $\eta_{\text {eff }}$, can be regarded as the major limits to interferometric precision [21,22,25,26,28,38,55,56,81-84]. Furthermore, it is customary to assume that the environmental noise and photonloss mechanism act identically and independently upon each probe mode [85], and that the environment is in a thermal state at a temperature determined by the mean photon number $n_{\mathrm{th}}$. Under these considerations, the light interfero- metric propagation is modified in the presence of decoherence as $\boldsymbol{S}(\varphi) \boldsymbol{V} \boldsymbol{S}^{T}(\varphi) \rightarrow \eta_{\text {loss }} \boldsymbol{S}(\varphi) \boldsymbol{V} \boldsymbol{S}^{T}(\varphi)+\left(1-\eta_{\text {loss }}\right)\left(1+n_{\text {th }}\right) \boldsymbol{I}_{N}$ [22,81,86,87]. Combining this result with Eqs. (9) and (10), we directly obtain

$$
\begin{gathered}
\langle\lambda(\varphi)\rangle=\sqrt{\eta_{\text {loss }}} \boldsymbol{S}(\varphi)\langle\boldsymbol{R}\rangle, \\
\boldsymbol{\sigma}(\varphi)=\left(1-\eta_{\mathrm{eff}}+\left(1-\eta_{\text {loss }}\right)\left(1+n_{\mathrm{th}}\right)\right) \boldsymbol{I}_{N} \\
+\underbrace{\eta_{\mathrm{eff}} \boldsymbol{\Sigma}+\eta_{\text {loss }} \boldsymbol{S}(\varphi) \boldsymbol{V} \boldsymbol{S}^{T}(\varphi)}_{\sigma_{\text {deco }}\left(\eta_{\text {loss }}, \eta_{\text {eff }}\right)},
\end{gathered}
$$

where the CV matrix $\sigma_{\text {deco }}$ solely regards photon-loss effects. By replacing (47) and (48) into the general equation (14) and doing some manipulation as illustrated in Sec. II C, we obtain a closed-form expression of the FI in presence of these decoherence effects, say $F_{\text {deco }}$, similar in structure to (18) [see Eq. (C35) in Appendix C3]. In the particular case we assume the propagation photon losses and nonunit efficiency contribute equally, i.e., $\eta_{\text {loss }}=\eta_{\text {eff }}=\eta$, the FI can be cast as follows:

$$
\begin{aligned}
F_{\mathrm{deco}}\left(\varphi, \eta, n_{\mathrm{th}}\right)= & \eta^{2} F(\varphi)-\eta\left\langle\boldsymbol{R}^{T}\right\rangle \boldsymbol{L}^{T} \boldsymbol{P}_{\varphi}^{T} \boldsymbol{\Sigma}_{\mathrm{deco}}^{-1}\left(\eta, n_{\mathrm{th}}\right) \boldsymbol{P}_{\varphi} \boldsymbol{L} \\
& \times\langle\boldsymbol{R}\rangle+\frac{1}{2} \operatorname{Tr}\left(\partial_{\varphi} \boldsymbol{\Sigma}_{\mathrm{deco}}^{-1}\left(\eta, n_{\mathrm{th}}\right) \partial_{\varphi} \boldsymbol{\sigma}_{\mathrm{deco}}(\eta)\right) \\
& -\left(1-\eta^{2}\right)\left(2+\frac{1}{2} \operatorname{Tr}\left(\left(\tilde{\boldsymbol{\Sigma}} \partial_{\varphi}\left(\boldsymbol{S} \boldsymbol{V} \boldsymbol{S}^{T}\right)\right)^{2}\right)\right),
\end{aligned}
$$

where $\boldsymbol{\Sigma}_{\text {deco }}\left(\eta, n_{\text {th }}\right)$ is a $2 N \times 2 N$ real, symmetric matrix [given by Eq. (C36)] that fully contains the influence owing to the environmental thermal noise. Recall $F(\varphi)$ denotes the FI in the ideal scenario.

Equation (49) manifests that the decoherence effects influence the phase resolution beyond a limiting constant factor of the phase sensitivity achievable in the ideal case [84]. Furthermore, this expression shows that the decoherence effects impact differently the phase sensitivity provided by distinct probe resources $[14,22,56]$ : while the third and last terms in the right-hand side vanish for input coherent states, the second term cancels for probe resources without an initial displacement. For instance, Eq. (49) indicates that thermal noise is especially detrimental for input displaced states, whilst the phase sensitivity due to coherent resources is apparently more tolerant to photon losses [22] [since the last term in (49) vanishes].

As a final remark, from Eq. (49) it is clear that the Gaussian interferometric schemes in presence of experimental imperfections cannot reach the HL; instead they could be able to improve upon the SNL for moderate values of $\eta$, as well as saturate the QCRB for quadrature measurements $[15,21,25,56,81]$. Rather than figuring out the strict homodyne measurement attaining the ultimate sensitivity given by the QFI in presence of photon loss and noise, from Eq. (49) one may be tempted to look for an alternative "optimal" Gaussian measurement scheme where optimal is understood in the sense that $F_{\text {deco }}$ eventually converges to $\eta^{2} \mathcal{F}$ instead (notice that $\mathcal{F}$ denotes the QFI in the ideal scenario). The latter yields an algebraic subsidiary condition as well, from which we may determine the corresponding optimal operating point. For instance, for the probe coherent scheme we find out that this is given by the formula $\sin \left(2 \varphi^{(x / p)}\right)=\mp\left[2(\eta / \tilde{\eta})^{2}-1\right]$ [see 
TABLE I. Summary of the phase sensitivity retrieved by the distinct Gaussian interferometric phase-estimation strategies involving an ideal homodyne detection, expressed in terms of the interferometer size $N$ and the input average photon number per mode $\bar{n}$. The particular choice of the probe Gaussian state and the interferometric scheme are specified in the first two columns. The third column illustrates the scaling of the FI with respect to $\bar{n}$ at a fixed value of the interferometer size $N$. The fourth column represents instead the scaling in terms of $N$ and for a given input intensity $\bar{n}$, whilst the fifth column determines which strategies are able to attain the QCRB. Accordingly, the SNL-type scaling in terms of the input intensity must be understood as $F \sim 4 \bar{n}$, whereas the SNL-type scaling is similarly defined as $F \sim 4 N$ in terms of the interferometer size.

\begin{tabular}{|c|c|c|c|c|}
\hline Input resources & $\begin{array}{l}\text { Interferometric } \\
\text { transformation }\end{array}$ & $\begin{array}{c}\text { Scaling } \\
\text { per mode energy }\end{array}$ & $\begin{array}{l}\text { Scaling with } \\
\text { interferometer size }\end{array}$ & QCRB \\
\hline $\begin{array}{l}\text { Coherent } \\
\left(s_{1}=s_{2}=1\right)\end{array}$ & QUMI & SNL & SNL & $\begin{array}{c}\text { Yes } \\
\text { See text around Eq. (27) }\end{array}$ \\
\hline $\begin{array}{l}\text { Single-mode squeezed vacuum } \\
\left(s_{1}=s_{2}=e^{-2 s^{\prime}}\right)\end{array}$ & Any & HL & Constant & $\begin{array}{c}\text { Yes } \\
\text { See Eq. }(40)\end{array}$ \\
\hline $\begin{array}{l}\text { One-mode squeezed } \otimes \text { coherent } \\
\left(s_{1}=e^{-2 s^{\prime}} \text { and } s_{2}=1\right)\end{array}$ & QUMI & Sub-SNL & Sub-SNL & $\begin{array}{c}\text { No (nearly optimal for } s^{\prime} \ll N \text { ) } \\
\text { See Eq. (37) }\end{array}$ \\
\hline Two-mode squeezed vacuum $(N=2)$ & 50:50 beam splitter & HL & & $\begin{array}{l}\text { Yes (for } \epsilon \neq 0 \text { ) } \\
\text { See Eq. }(46)\end{array}$ \\
\hline
\end{tabular}

Eq. (C37)], with

$$
\tilde{\eta}^{2}=\eta^{2}+\frac{\left(2+n_{\mathrm{th}}\right)(1-\eta)}{\eta+(1-\eta) n_{\mathrm{th}}-2},
$$

which significantly differs from the ideal scenario (i.e., $\left.\varphi_{\mathrm{opt}}^{(x / p)}=\mp \pi / 4\right)$. This manifests that the experimental imperfections substantially influence the optimal working point besides the ultimate sensitivity.

\section{OUTLOOK AND CONCLUDING REMARKS}

In this paper we have presented a theoretical framework to explore the metrological potential of generic Gaussian interferometric schemes accessible with current photonic technology. Our treatment proves convenient to address the optimal phase-estimation scheme and operating point: in particular, we recover the vast majority of previous well-known results in the single- and two-mode Gaussian metrology scenarios. In Table I, we summarize the phase sensitivity provided by the choice of different input states and interferometric schemes in the finite energetic regime. To a large extent this table contains most of the previous results related to Gaussian phase resolution in the absence of photon loss and for perfect detection schemes [21,22,26,38].

Interestingly, input coherent resources were shown to outperform the probe nonclassical states used in previous QUMI-based phase-estimation proposals. Moreover, our analysis revealed that in the low-intensity regime (e.g., when the squeezing parameter is small compared to the interferometer size $N$ ) the QUMI architecture along with probe singlemode squeezed states is unable to provide a real metrological advantage with respect to the best classical strategy for a large $N$.

Additionally, we also developed a polychromatic version of the well-established MZI setup endowed with probe twomode nondegenerate squeezed-vacuum states. We show that this setup can significantly improve the resolution-energy tradeoff with optimal (ideal) quadrature measurements. Also our treatment is a versatile approach to address the impact of experimental imperfections on the phase sensitivity unlike the analysis based on the complex SLD: e.g., we show that the optimal working point associated to coherent resources is significantly shifted by both the photon losses and the nonunitefficiency detection.

Remarkably, the recent developments on the fabrication and manipulation of integrated photonic circuits $[42,43,88]$ makes them more resilient to phase stability, or photon losses and noise effects, which opens new avenues to implement higher sophisticated phase-estimation experiments with relatively little effort [39] (e.g., endowed with current photon sources and measurement detection schemes). In particular this prospect highlights the demand for further theoretical tools to enable us to explore its feasible metrological power. In this sense, the present treatment could render a valuable theoretical support to envisage a new series of experiments in the realm of quantum phase estimation.

\section{ACKNOWLEDGMENTS}

This material is based upon work supported by AFOSR Grant No. FA2386-18-1-4019. The authors also acknowledge support from the Spanish Ministry of Economic Affairs and Digital Transformation Project No. PGC2018-094792-B-100 (Ministerio de Ciencia, Innovación, y Universidades-Agencia Estatal de Investigación-Regional Development European Funds, European Union).

\section{APPENDIX A: BASICS OF PHASE ESTIMATION}

In this section we briefly sketch the derivation of the general expressions (14) and (17) by using results from matrix analysis theory $[64,65]$ and the matrix identities (2) and (7) just relying on the interested set of probe isothermal Gaussian states. We start from the formal definition of the FI, which reads $[8,9,15]$

$$
F(\varphi)=\int d^{2 m} \lambda \frac{1}{p(\lambda \mid \varphi)}\left(\frac{\partial p(\lambda \mid \varphi)}{\partial \varphi}\right)^{2} .
$$

Thanks to the fact that the probability distribution characterizing the Gaussian phase-estimation scheme is a Gaussian 
function, the result of the integral involved in (A1) is a Gaussian function as well. This can be seen more clearly once the derivative of the probability distribution is computed, i.e.,

$$
\left.\frac{\partial p(\lambda \mid \varphi)}{\partial \varphi}=\frac{1}{2} p(\lambda \mid \varphi)\left((\lambda-\langle\lambda\rangle)^{T} \sigma^{-1} \partial_{\varphi} \sigma \sigma^{-1}(\lambda-\langle\lambda\rangle)+2(\lambda-\langle\lambda\rangle)^{T} \sigma^{-1} \partial_{\varphi}\langle\lambda\rangle\right)-\operatorname{Tr}\left(\sigma^{-1} \partial_{\varphi} \sigma\right)\right),
$$

where the last term of the right-hand side appears due to the dependence of the probability distribution normalization constant with the desired phase shift [64]. Hence, one may realize that the classical Fisher information (A1) reduces to carry out the integral of a quadratic polynomial (in the variable $\lambda$ ) weighted by $p(\lambda \mid \varphi)$. After substituting Eq. (A2) in (A1), it is convenient to swap from $p(\lambda \mid \varphi)$ to a zero-mean Gaussian probability distribution $p(\tilde{\lambda} \mid \varphi)$, with the CV $\sigma$, by making the change of variables $\tilde{\lambda}=\sigma^{-1}(\lambda-\langle\lambda\rangle)$. Upon doing this, we obtain

$$
\begin{aligned}
F(\varphi)= & \frac{1}{4} \int d^{2 m} \tilde{\lambda} p(\tilde{\lambda} \mid \varphi)\left(\left(\operatorname{Tr}\left(\sigma^{-1} \partial_{\varphi} \sigma\right)\right)^{2}-2 \operatorname{Tr}\left(\sigma^{-1} \partial_{\varphi} \sigma\right)\left(\tilde{\lambda}^{T} \partial_{\varphi} \sigma \tilde{\lambda}-2 \partial_{\varphi}\left\langle\lambda^{T}\right\rangle \tilde{\lambda}\right)\right. \\
& \left.+\left(\left(\tilde{\lambda}^{T} \partial_{\varphi} \sigma \tilde{\lambda}\right)\left(\tilde{\lambda}^{T} \partial_{\varphi} \sigma \tilde{\lambda}\right)+4\left(\partial_{\varphi}\langle\lambda\rangle \tilde{\lambda}\right)\left(\partial_{\varphi}\langle\lambda\rangle \tilde{\lambda}\right)-4\left(\partial_{\varphi}\langle\lambda\rangle \tilde{\lambda}\right)\left(\tilde{\lambda}^{T} \partial_{\varphi} \sigma \tilde{\lambda}\right)\right)\right) .
\end{aligned}
$$

Since $p(\tilde{\lambda} \mid \varphi)$ is centered with respect to the origin $\tilde{\lambda}=0$, the contribution coming from the linear and third-order terms in the right-hand side of (A3) must cancel. The rest of the contributions can be computed by using the following identity for multivariate Gaussian integrals [64]:

$$
\int d^{2 m} \lambda|\lambda|^{4} \exp \left(-\frac{1}{2}(\lambda-\langle\lambda\rangle) \sigma^{-1}(\lambda-\langle\lambda\rangle)\right)=|\langle\lambda\rangle|^{4}+4\left\langle\lambda^{T}\right\rangle \sigma\langle\lambda\rangle+\operatorname{Tr}^{2}(\sigma)+2 \operatorname{Tr}(\sigma)|\langle\lambda\rangle|^{2}+2 \operatorname{Tr}\left(\sigma \sigma^{T}\right),
$$

with $|\boldsymbol{x}|$ denoting the usual Euclidean norm of a $2 m$ dimensional vector $\boldsymbol{x}$. After substituting the result (A4) in (A3), we obtain an expression with several terms involving the derivative of $\sigma$; these terms can be further simplified by using the matrix identity $\partial_{\varphi} \sigma^{-1}=-\sigma^{-1} \partial_{\varphi} \sigma \sigma^{-1}$ [64]. This finally leads us to Eq. (14).

Now we turn the attention to the formula (17) of the QFI by virtue of the phase generator $\boldsymbol{U}_{N}(\varphi)=\boldsymbol{U}(\varphi) \oplus \boldsymbol{I}_{N-1}$ with

$$
\boldsymbol{U}(\varphi)=\left(\begin{array}{cc}
\cos \varphi & \sin \varphi \\
-\sin \varphi & \cos \varphi
\end{array}\right)
$$

This is worked out from the general expression of the QFI valid for any pure or mixed single-mode Gaussian state provided in $[2,26,44]$. The latter takes the following form for the set $\mathcal{G}\left(1, \bar{n}_{t}\right)$ of interesting states and the phase-shift generator $(\mathrm{A} 5)$ :

$$
\mathcal{F}=\left\langle\boldsymbol{R}_{1}^{\prime T}\right\rangle \partial_{\varphi} \boldsymbol{U}^{T}(\varphi) \boldsymbol{U}(\varphi) \boldsymbol{V}_{1}^{\prime-1} \boldsymbol{U}^{T}(\varphi) \partial_{\varphi} \boldsymbol{U}(\varphi)\left\langle\boldsymbol{R}_{1}^{\prime}\right\rangle-\frac{1}{2\left(1+\left(2 \bar{n}_{t}+1\right)^{-2}\right)} \operatorname{Tr}\left(\partial_{\varphi}\left(\boldsymbol{U}^{T}(\varphi) \boldsymbol{V}_{1}^{\prime-1} \boldsymbol{U}(\varphi)\right)\left(\partial_{\varphi}\left(\boldsymbol{U}^{T}(\varphi) \boldsymbol{V}_{1}^{\prime} \boldsymbol{U}(\varphi)\right)\right)\right.
$$

By replacing the matrix identity (2), we obtain upon some manipulation

$$
\begin{aligned}
\mathcal{F}= & \frac{1}{\left(2 \bar{n}_{t}+1\right)^{2}}\left\langle\boldsymbol{R}_{1}^{\prime T}\right\rangle \partial_{\varphi} \boldsymbol{U}^{T}(\varphi) \boldsymbol{U}(\varphi) \boldsymbol{V}_{1}^{\prime} \boldsymbol{U}^{T}(\varphi) \partial_{\varphi} \boldsymbol{U}(\varphi)\left\langle\boldsymbol{R}_{1}^{\prime}\right\rangle+\frac{\left(2 \bar{n}_{t}+1\right)^{-2}}{2\left(1+\left(2 \bar{n}_{t}+1\right)^{-2}\right)} \operatorname{Tr}\left(\partial_{\varphi}\left(\boldsymbol{J} \boldsymbol{U}^{T}(\varphi) \boldsymbol{V}_{1}^{\prime} \boldsymbol{U}(\varphi)\right)^{2}\right) \\
= & \frac{1}{\left(2 \bar{n}_{t}+1\right)^{2}}\left(\left\langle\boldsymbol{R}_{1}^{\prime T}\right\rangle \partial_{\varphi} \boldsymbol{U}^{T}(\varphi) \boldsymbol{U}(\varphi) \boldsymbol{V}_{1}^{\prime} \boldsymbol{U}^{T}(\varphi) \partial_{\varphi} \boldsymbol{U}(\varphi)\left\langle\boldsymbol{R}_{1}^{\prime}\right\rangle+\frac{1}{1+\left(2 \bar{n}_{t}+1\right)^{-2}}\left(\operatorname{Tr}\left(\left(\boldsymbol{J} \partial_{\varphi} \boldsymbol{U}^{T}(\varphi) \boldsymbol{V}_{1}^{\prime} \boldsymbol{U}(\varphi)\right)^{2}\right)\right.\right. \\
& \left.\left.-\left(2 \bar{n}_{t}+1\right)^{2} \operatorname{Tr}\left(\partial_{\varphi} \boldsymbol{U}^{T}(\varphi) \partial_{\varphi} \boldsymbol{U}(\varphi)\right)\right)\right)
\end{aligned}
$$

which after substituting $\partial_{\varphi} \boldsymbol{U}(\varphi)=\boldsymbol{J} \boldsymbol{U}(\varphi)$ leads to the expression (17). Notice that in the pure case (i.e., $\left.\bar{n}_{t}=0\right)$ the expression (17) identically coincides with the result independently obtained from the standard expression of the QFI $\mathcal{F}=4(\Delta \hat{H})^{2}$.

\section{APPENDIX B: GAUSSIAN PHASE ESTIMATION}

In this Appendix we extensively illustrate the derivation of Eq. (18) appearing in Sec. II C. We first express the inverse of (10) in terms of the CV matrix $\sigma_{S}$ by means of the Woodbury identity $[64,65]$, that is

$$
\sigma^{-1}=\sigma_{S}^{-1}-\sigma_{S}^{-1} S_{S A}\left(V_{A}^{-1}+S_{S A}^{T} \sigma_{S}^{-1} S_{S A}\right)^{-1} S_{S A}^{T} \sigma_{S}^{-1},
$$

which always holds as $\boldsymbol{V}_{A}^{-1}+\boldsymbol{S}_{S A}^{T} \boldsymbol{\sigma}_{S}^{-1} \boldsymbol{S}_{S A}$ is expected to be an invertible matrix. Notice that we have omitted the explicit dependence of the matrices with $\varphi$ for the sake of clarity. The above identity allows us to separate the FI contribution in (14) that is completely independent of the ancilla CV matrix. This yields the expression (23). In other words, we gather together in $F_{S}(\varphi)$ all dependence in $\sigma_{S}$. Since $\boldsymbol{\Sigma}_{S}^{-1}+\left(\boldsymbol{S}_{S}^{T}\right)^{-1} \boldsymbol{V}_{S}^{-1} \boldsymbol{S}_{S}^{-1}$ 
must be an invertible matrix as well (see remark 2.16.21 in [65]), we can employ again the Woodbury identity in order to express the $\sigma_{S}^{-1}$ in terms of $\boldsymbol{V}_{S}^{-1}$, which is

$$
\begin{aligned}
\boldsymbol{\sigma}_{S}^{-1}= & \left(\boldsymbol{S}_{S}^{T}\right)^{-1} \boldsymbol{V}_{S}^{-1} \boldsymbol{S}_{S}^{-1}-\left(\boldsymbol{S}_{S}^{T}\right)^{-1} \boldsymbol{V}_{S}^{-1} \boldsymbol{S}_{S}^{-1} \\
& \times\left(\boldsymbol{\Sigma}_{S}^{-1}+\left(\boldsymbol{S}_{S}^{T}\right)^{-1} \boldsymbol{V}_{S}^{-1} \boldsymbol{S}_{S}^{-1}\right)^{-1}\left(\boldsymbol{S}_{S}^{T}\right)^{-1} \boldsymbol{V}_{S}^{-1} \boldsymbol{S}_{S}^{-1}
\end{aligned}
$$

Replacing this result in the obtained expression for $F_{S}(\varphi)$, we arrive to

$$
\begin{aligned}
F_{S}(\varphi)= & \left\langle\boldsymbol{R}_{S}^{T}\right\rangle \partial_{\varphi} \boldsymbol{S}_{S}^{T}\left(\boldsymbol{S}_{S}^{T}\right)^{-1} \boldsymbol{V}_{S}^{-1} \boldsymbol{S}_{S}^{-1} \partial_{\varphi} \boldsymbol{S}_{S}\left\langle\boldsymbol{R}_{S}\right\rangle \\
& -\frac{1}{2} \operatorname{Tr}\left(\partial_{\varphi}\left(\left(\boldsymbol{S}_{S}^{T}\right)^{-1} \boldsymbol{V}_{S}^{-1} \boldsymbol{S}_{S}^{-1}\right) \partial_{\varphi}\left(\boldsymbol{S}_{S} \boldsymbol{V}_{S} \boldsymbol{S}_{S}^{T}\right)\right)-F_{\text {Meas }}(\varphi),
\end{aligned}
$$

where we have identified $F_{\text {Meas }}(\varphi)$ as the residual contribution given by Eq. (19). We can further simplify (B3) by substituting the following identities satisfied by the inverse of the sub-block matrices of $\boldsymbol{S}$ (see proposition 2.8.7 in [65]):

$$
\begin{gathered}
S_{S}^{-1}=S_{S}^{T}-\Delta S_{S}, \\
\left(S_{S}^{T}\right)^{-1}=S_{S}-\Delta S_{S}^{T},
\end{gathered}
$$

with

$$
\Delta \boldsymbol{S}_{S}=\boldsymbol{S}_{S}^{-1} \boldsymbol{S}_{S A}\left(\boldsymbol{S} / \boldsymbol{S}_{S}\right)^{-1} \boldsymbol{S}_{A S} \boldsymbol{S}_{S}^{-1},
$$

where $\boldsymbol{S} / \boldsymbol{S}_{S}$ stands for the Schur complement of $\boldsymbol{S}_{S}$ in $\boldsymbol{S}$, i.e., $\boldsymbol{S} / \boldsymbol{S}_{S}=\boldsymbol{S}_{A}-\boldsymbol{S}_{A S} \boldsymbol{S}_{S}^{-1} \boldsymbol{S}_{S A}$, which is nonsingular for a realistic transformation $S$. By plugging the relations (B4) into (B3), after some tedious manipulation we obtain

$$
\begin{aligned}
F_{S}(\varphi)= & \left\langle\boldsymbol{R}_{S}^{T}\right\rangle \partial_{\varphi} \boldsymbol{S}_{S}^{T} \boldsymbol{S}_{S} \boldsymbol{V}_{S}^{-1} \boldsymbol{S}_{S}^{T} \partial_{\varphi} \boldsymbol{S}_{S}\left\langle\boldsymbol{R}_{S}\right\rangle \\
& -\frac{1}{2} \operatorname{Tr}\left(\partial_{\varphi}\left(\boldsymbol{S}_{S} \boldsymbol{V}_{S}^{-1} \boldsymbol{S}_{S}^{T}\right) \partial_{\varphi}\left(\boldsymbol{S}_{S} \boldsymbol{V}_{S} \boldsymbol{S}_{S}^{T}\right)\right)+\tilde{F}_{\text {Int }}(\varphi) \\
& -F_{\text {Meas }}(\varphi),
\end{aligned}
$$

where

$$
\tilde{F}_{\text {Int }}(\varphi)=F_{\text {Int }}(\varphi)-\operatorname{Tr}\left(\partial_{\varphi} \boldsymbol{S}_{S}^{T} \partial_{\varphi} \boldsymbol{S}_{S} \boldsymbol{V}_{S}^{-1} \boldsymbol{S}_{S A} \boldsymbol{S}_{S A}^{T} \boldsymbol{V}_{S}\right),
$$

and $F_{\text {Int }}(\varphi)$ is given by

$$
\begin{aligned}
F_{\text {Int }}(\varphi)= & \operatorname{Tr}\left(\partial_{\varphi} \boldsymbol{S}_{S}^{T} \partial_{\varphi} \boldsymbol{S}_{S} \boldsymbol{V}_{S}^{-1} \boldsymbol{S}_{S A} \boldsymbol{S}_{S A}^{T} \boldsymbol{V}_{S}\right)-2\left\langle\boldsymbol{R}_{S}^{T}\right\rangle \partial_{\varphi} \boldsymbol{S}_{S}^{T} \boldsymbol{S}_{S} \boldsymbol{V}_{S}^{-1} \Delta \boldsymbol{S}_{S} \partial_{\varphi} \boldsymbol{S}_{S}\left\langle\boldsymbol{R}_{S}\right\rangle \\
& +\left\langle\boldsymbol{R}_{S}^{T}\right\rangle \partial_{\varphi} \boldsymbol{S}_{S}^{T} \Delta \boldsymbol{S}_{S}^{T} \boldsymbol{V}_{S}^{-1} \Delta \boldsymbol{S}_{S} \partial_{\varphi} \boldsymbol{S}_{S}\left\langle\boldsymbol{R}_{S}\right\rangle+\frac{1}{2} \operatorname{Tr}\left(\partial_{\varphi}\left(2 \boldsymbol{S}_{S} \boldsymbol{V}_{S}^{-1} \Delta \boldsymbol{S}_{S}-\Delta \boldsymbol{S}_{S}^{T} \boldsymbol{V}_{S}^{-1} \Delta \boldsymbol{S}_{S}\right) \partial_{\varphi}\left(\boldsymbol{S}_{S} \boldsymbol{V}_{S} \boldsymbol{S}_{S}^{T}\right)\right) .
\end{aligned}
$$

Now we substitute the inverse matrix $\boldsymbol{V}_{S}^{-1}$ in (B7) according to the symplecticlike identity (2), and then we use the relation (7) to cast Eq. (B7) in the following form:

$$
\begin{aligned}
F_{S}(\varphi)= & \frac{1}{\left(2 \bar{n}_{t}+1\right)^{2}}\left(\left\langle\boldsymbol{R}_{S}^{T}\right\rangle \partial_{\varphi}\left(\boldsymbol{J}_{m} \boldsymbol{S}_{S}\right)^{T} \boldsymbol{S}_{S} \boldsymbol{V}_{S} \boldsymbol{S}_{S}^{T} \partial_{\varphi}\left(\boldsymbol{J}_{m} \boldsymbol{S}_{S}\right)\left\langle\boldsymbol{R}_{S}\right\rangle+\frac{1}{2} \operatorname{Tr}\left(\left(\partial_{\varphi}\left(\boldsymbol{J}_{m} \boldsymbol{S}_{S} \boldsymbol{V}_{S} \boldsymbol{S}_{S}^{T}\right)\right)^{2}\right)\right) \\
& +\tilde{F}_{\text {Int }}(\varphi)-F_{\text {Meas }}(\varphi) .
\end{aligned}
$$

Let us now focus the attention on the trace term of Eq. (B10). This term can be simplified as follows:

$$
\begin{aligned}
\operatorname{Tr}\left(\left(\partial_{\varphi}\left(\boldsymbol{J}_{m} \boldsymbol{S}_{S} \boldsymbol{V}_{S} \boldsymbol{S}_{S}^{T}\right)\right)^{2}\right)= & \operatorname{Tr}\left(\left(\boldsymbol{J}_{m} \partial_{\varphi} \boldsymbol{S}_{S} \boldsymbol{V}_{S} \boldsymbol{S}_{S}^{T}+\boldsymbol{J}_{m} \boldsymbol{S}_{S} \boldsymbol{V}_{S} \partial_{\varphi} \boldsymbol{S}_{S}^{T}\right)^{2}\right) \\
= & 2 \operatorname{Tr}\left(\left(\boldsymbol{J}_{m} \partial_{\varphi} \boldsymbol{S}_{S} \boldsymbol{V}_{S} \boldsymbol{S}_{S}^{T}\right)^{2}\right)+2 \operatorname{Tr}\left(\partial_{\varphi} \boldsymbol{S}_{S} \boldsymbol{J}_{m} \boldsymbol{V}_{S} \boldsymbol{J}_{m} \boldsymbol{S}_{S}^{T} \boldsymbol{S}_{S} \boldsymbol{V}_{S} \partial_{\varphi} \boldsymbol{S}_{S}^{T}\right) \\
= & 2 \operatorname{Tr}\left(\left(\partial_{\varphi}\left(\boldsymbol{J}_{m} \boldsymbol{S}_{S}\right) \boldsymbol{V}_{S} \boldsymbol{S}_{S}^{T}\right)^{2}\right)-2(2 \bar{n}+1)^{2}\left(\operatorname{Tr}\left(\partial_{\varphi} \boldsymbol{S}_{S}^{T} \partial_{\varphi} \boldsymbol{S}_{S}\right)\right. \\
& \left.-\operatorname{Tr}\left(\partial_{\varphi} \boldsymbol{S}_{S}^{T} \partial_{\varphi} \boldsymbol{S}_{S} \boldsymbol{V}_{S}^{-1} \boldsymbol{S}_{S A} \boldsymbol{S}_{S A}^{T} \boldsymbol{V}_{S}\right)\right),
\end{aligned}
$$

where once again we have made use of the linear properties of the trace, as well as the identities (2) and (6). Replacing the result (B11) in Eq. (B10) directly returns the expression

$$
\begin{aligned}
F_{S}(\varphi)= & \left(\left\langle\boldsymbol{R}_{S}^{T}\right\rangle \partial_{\varphi}\left(\boldsymbol{J}_{m} \boldsymbol{S}_{S}\right)^{T} \boldsymbol{S}_{S} \boldsymbol{V}_{S} \boldsymbol{S}_{S}^{T} \partial_{\varphi}\left(\boldsymbol{J}_{m} \boldsymbol{S}_{S}\right)\left\langle\boldsymbol{R}_{S}\right\rangle+\operatorname{Tr}\left(\left(\partial_{\varphi}\left(\boldsymbol{J}_{m} \boldsymbol{S}_{S}\right) \boldsymbol{V}_{S} \boldsymbol{S}_{S}^{T}\right)^{2}\right)\right) \frac{1}{\left(2 \bar{n}_{t}+1\right)^{2}} \\
& -\operatorname{Tr}\left(\partial_{\varphi} \boldsymbol{S}_{S}^{T} \partial_{\varphi} \boldsymbol{S}_{S}\right)-F_{\text {Meas }}(\varphi)+F_{\text {Int }}(\varphi),
\end{aligned}
$$

after rearranging the contribution $\operatorname{Tr}\left(\partial_{\varphi} \boldsymbol{S}_{S}^{T} \partial_{\varphi} \boldsymbol{S}_{S} \boldsymbol{V}_{S}^{-1} \boldsymbol{S}_{S A} \boldsymbol{S}_{S A}^{T} \boldsymbol{V}_{S}\right)$ into the definition of $F_{\text {Int }}(\varphi)$. One can proceed by noticing from (A5) that $\partial_{\varphi} \boldsymbol{U}(\varphi)=\boldsymbol{U}(\varphi) \boldsymbol{J}$. The latter combined with Eq. (5) directly yields

$$
\partial_{\varphi} \boldsymbol{S}_{S}(\varphi)=\boldsymbol{P}_{\varphi} \boldsymbol{J}_{m} \boldsymbol{L}_{S}
$$

where $\boldsymbol{P}_{\varphi}=\boldsymbol{U}(\varphi) \oplus \mathbf{0}_{m-1}$, which is a $2 m \times 2 m$ projection matrix (i.e., $\boldsymbol{P}_{\varphi} \boldsymbol{P}_{\varphi}^{T}=\boldsymbol{P}_{\varphi}^{T} \boldsymbol{P}_{\varphi}=\boldsymbol{I}_{1} \oplus \mathbf{0}_{m-1}$ as well as $\left.\boldsymbol{J}_{m}^{T} \boldsymbol{P}_{\varphi} \boldsymbol{J}_{m}=\boldsymbol{P}_{\varphi}\right)$. Here $\mathbf{0}_{m-1}$ stands for the $2(m-1) \times 2(m-$ 1) null matrix (i.e., all its entries are zero), so that the effect of $\boldsymbol{P}_{\varphi}$ through the subsequent computation is to drop the explicit dependence with the slice of matrix that is not supported by the phase space of the phase-shifted mode $\left(\hat{q}_{1}, \hat{p}_{1}\right)$ : for 
instance, for value $\varphi=0,\left\langle\boldsymbol{R}_{S}\right\rangle$ and $\boldsymbol{V}_{S}$ get projected into the displacement vector $\left\langle\boldsymbol{R}_{1}^{\prime}\right\rangle$ and the CV matrix $\boldsymbol{V}_{1}^{\prime}$ of the first probe mode immediately before undergoing the phase rotation, that is $\left\langle\boldsymbol{R}_{1}^{\prime}\right\rangle=\boldsymbol{P}_{0} \boldsymbol{L}_{S}\left\langle\boldsymbol{R}_{S}\right\rangle$ and $\boldsymbol{V}_{1}^{\prime}=\boldsymbol{P}_{0} \boldsymbol{L}_{S} \boldsymbol{V}_{S} \boldsymbol{L}_{S}^{T} \boldsymbol{P}_{0}^{T}$. By virtue of the latter, after some manipulation replacing Eq. (B13) in (B12), one gets

$$
\begin{aligned}
F_{S}(\varphi)= & \frac{1}{\left(2 \bar{n}_{t}+1\right)^{2}}\left(\left\langle\boldsymbol{R}_{1}^{\prime T}\right| \boldsymbol{V}_{1}^{\prime}\left\langle\boldsymbol{R}_{1}^{\prime}\right\rangle+\operatorname{Tr}\left(\boldsymbol{V}_{1}^{\prime} \boldsymbol{V}_{1}^{\prime}\right)\right) \\
& -F_{\text {Meas }}(\varphi)+F_{\text {Int }}(\varphi)-\operatorname{Tr}\left(\boldsymbol{P}_{0} \boldsymbol{L}_{S} \boldsymbol{L}_{S}^{T}\right),
\end{aligned}
$$

from which one can identify the QFI characteristic of the $m$ mode probe system upon close inspection. By conveniently manipulating (B14) once plunged into (23), we arrive at the desired expression (18) for the FI.

In the particular case of nonassisted phase-estimation schemes and pure input Gaussian states, the expression (18) boils down to (28). In this scenario, the aforementioned auxiliary matrix $\tilde{\boldsymbol{\Sigma}}$ further becomes $\boldsymbol{S} \boldsymbol{V}^{-1} \boldsymbol{S}^{T}\left(\boldsymbol{\Sigma}^{-1}+\right.$ $\left.S V^{-1} S^{T}\right)^{-1} S V^{-1} S^{T}$, so that the measurement contribution $F_{\text {Meas }}$, given by Eq. (19), can be substantially simplified as well. More specifically, by substituting this observation we obtain the first term in the right-hand side of (29), whereas the second terms may be further simplified by using the symplecticlike identities for $\boldsymbol{\Sigma}, \boldsymbol{S}(\varphi)$, and $\boldsymbol{V}$ as before (as well as $\left.\partial_{\varphi} \boldsymbol{A}^{-1}=-\boldsymbol{A}^{-1} \partial_{\varphi} \boldsymbol{A} \boldsymbol{A}^{-1}\right)$ :

$$
\begin{aligned}
\operatorname{Tr}\left(\partial_{\varphi}\right. & \left.\tilde{\boldsymbol{\Sigma}} \partial_{\varphi}\left(\boldsymbol{S} \boldsymbol{V} \boldsymbol{S}^{T}\right)\right) \\
= & \operatorname{Tr}\left(\partial_{\varphi}\left(\boldsymbol{\Sigma}^{-1}+\boldsymbol{S} \boldsymbol{V}^{-1} \boldsymbol{S}^{T}\right)^{-1} \boldsymbol{S} \boldsymbol{V}^{-1} \boldsymbol{S}^{T} \partial_{\varphi}\left(\boldsymbol{S} \boldsymbol{V} \boldsymbol{S}^{T}\right) \boldsymbol{S} \boldsymbol{V}^{-1} \boldsymbol{S}^{T}\right) \\
& +2 \operatorname{Tr}\left(\partial_{\varphi}\left(\boldsymbol{S} \boldsymbol{V}^{-1} \boldsymbol{S}^{T}\right)\left(\boldsymbol{\Sigma}^{-1}+\boldsymbol{S} \boldsymbol{V}^{-1} \boldsymbol{S}^{T}\right)^{-1}\left(\boldsymbol{S} \boldsymbol{V}^{-1} \boldsymbol{S}^{T}\right)\right. \\
& \left.\times \partial_{\varphi}\left(\boldsymbol{S} \boldsymbol{V} \boldsymbol{S}^{T}\right)\right) \\
= & -\operatorname{Tr}\left(\boldsymbol{J}_{N}^{T} \partial_{\varphi}\left(\boldsymbol{\Sigma}+\boldsymbol{S} \boldsymbol{V} \boldsymbol{S}^{T}\right)^{-1} \boldsymbol{J}_{N} \partial_{\varphi}\left(\boldsymbol{S} \boldsymbol{V}^{-1} \boldsymbol{S}^{T}\right)\right) \\
& +2 \operatorname{Tr}\left(\left(\boldsymbol{\Sigma}^{-1}+\boldsymbol{S} \boldsymbol{V}^{-1} \boldsymbol{S}^{T}\right)^{-1} \boldsymbol{J}_{N}\left(\boldsymbol{S} \boldsymbol{V} \boldsymbol{S}^{T}\right) \boldsymbol{J}_{N}^{T} \partial_{\varphi}\left(\boldsymbol{S} \boldsymbol{V} \boldsymbol{S}^{T}\right)\right. \\
& \left.\times \boldsymbol{J}_{N} \partial_{\varphi}\left(\boldsymbol{S} \boldsymbol{V} \boldsymbol{S}^{T}\right) \boldsymbol{J}_{N}^{T}\right) \\
= & \operatorname{Tr}\left(\left(\boldsymbol{\Sigma}+\boldsymbol{S} \boldsymbol{V} \boldsymbol{S}^{T}\right)^{-1} \partial_{\varphi}\left(\boldsymbol{S} \boldsymbol{V} \boldsymbol{S}^{T}\right)\left(\boldsymbol{\Sigma}+\boldsymbol{S} \boldsymbol{V} \boldsymbol{S}^{T}\right)^{-1} \partial_{\varphi}\right. \\
& \left.\times\left(\boldsymbol{S} \boldsymbol{V} \boldsymbol{S}^{T}\right)\right)-2 \operatorname{Tr}\left(\left(\boldsymbol{\Sigma}+\boldsymbol{S} \boldsymbol{V} \boldsymbol{S}^{T}\right)^{-1}\left(\boldsymbol{S} \boldsymbol{V} \boldsymbol{S}^{T}\right)\right. \\
& \left.\times \partial_{\varphi}\left(\boldsymbol{J}_{N} \boldsymbol{S} \boldsymbol{V} \boldsymbol{S}^{T}\right) \partial_{\varphi}\left(\boldsymbol{J}_{N} \boldsymbol{S} \boldsymbol{V} \boldsymbol{S}^{T}\right)\right),
\end{aligned}
$$

where once again we have employed the linearity properties of the trace and $\boldsymbol{J}_{N}=-\boldsymbol{J}_{N}^{T}$. By substituting (B15) in (19), it is clear to see that we arrive at the desired expression (29) for the measurement contribution.

\section{APPENDIX C: $N$-MODE HOMODYNE DETECTION WITH INPUT COHERENT STATES AND WITHOUT AN ANCILLA SYSTEM}

\section{Explicit expressions from Sec. III A}

In this section we provide the explicit form corresponding to the particular QUMI transformation studied in Sec. II C, as well as the functions and matrices involved in the expressions from (32) to (38) appearing in Sec. III B.

In the phase-space notation, the transformation of the first probe mode due to any interferometric operation can be compactly expressed as follows:

$$
\boldsymbol{R}_{1} \rightarrow \sum_{i=1}^{N} V_{1 i} \boldsymbol{R}_{i} \quad \text { with } \quad \boldsymbol{R}_{i}=\left(x_{i}, p_{i}\right)
$$

where $V_{1 i}$ is determined by the unitary evolution describing the interferometric operation. As stated in Sec. IV of [46], the family of QUMI transformations is formally characterized by the constraint $\left|V_{1 j}\right|=1 / \sqrt{N}$ for $j=1, \ldots, N$ [see discussion below Eq. (5)]. In particular, we will focus the attention on the subset of QUMI transformations which satisfies $V_{1 j}=1 / \sqrt{N}$. For this subset and for input coherent states with $\left\langle\boldsymbol{R}_{i}\right\rangle=\left(\sqrt{2 \bar{n}_{c}}, \sqrt{2 \bar{n}_{c}}\right)$ for $i \in[1, N]$, the average photon number of the first probe mode after transformation is equal to the average number of input photons, as mentioned in the discussion around Eq. (26).

The interesting subset of QUMI schemes is provided by the following prescription [46]: The probe $N$ mode sequentially interferes with all the remaining input modes, and the transmissivity between the probe $N$ and $j$ mode is given by $\tau=1-1 / j$ (notice that $\sqrt{1-\tau}$ retrieves the transmissivity amplitude in the notation of [46]). Based on this prescription, we found out that the orthogonal matrix, say $\boldsymbol{L}_{\mathrm{QUMI}}$, associated to a simple QUMI architecture takes the form

$$
\boldsymbol{L}_{\mathrm{QUMI}}=\left(\begin{array}{cccccccc}
\sqrt{\frac{1}{N}} & 0 & \sqrt{\frac{1}{N}} & 0 & \sqrt{\frac{1}{N}} & 0 & \cdots & 0 \\
0 & \sqrt{\frac{1}{N}} & 0 & \sqrt{\frac{1}{N}} & 0 & \sqrt{\frac{1}{N}} & \cdots & \sqrt{\frac{1}{N}} \\
-\sqrt{\frac{N-1}{N}} & 0 & \sqrt{\frac{1}{N(N-1)}} & 0 & \sqrt{\frac{1}{N(N-1)}} & 0 & \cdots & 0 \\
0 & -\sqrt{\frac{N-1}{N}} & 0 & \sqrt{\frac{1}{N(N-1)}} & 0 & \sqrt{\frac{1}{N(N-1)}} & \cdots & \sqrt{\frac{1}{N(N-1)}} \\
0 & 0 & -\sqrt{\frac{N-2}{N-1}} & 0 & \sqrt{\frac{1}{(N-1)(N-2)}} & 0 & \cdots & 0 \\
0 & 0 & 0 & -\sqrt{\frac{N-2}{N-1}} & 0 & \sqrt{\frac{1}{(N-1)(N-2)}} & \cdots & \sqrt{\frac{1}{(N-1)(N-2)}} \\
0 & 0 & 0 & \cdots & & \vdots & & \vdots \\
0 & 0 & 0 & \cdots & -\sqrt{\frac{1}{2}} & 0 & \sqrt{\frac{1}{2}} & 0 \\
0 & 0 & 0 & \cdots & 0 & -\sqrt{\frac{1}{2}} & 0 & \sqrt{\frac{1}{2}}
\end{array}\right) .
$$


Note that $\boldsymbol{L}_{\mathrm{QUMI}}$ satisfies the condition which defines the QUMI family of transformations: all matrix elements on the first row are identical to $1 / \sqrt{N}$. Let us briefly sketch how to obtain the result (C2) by following a mathematical induction procedure: we initially prove that $\boldsymbol{L}_{\mathrm{QUMI}}$ with $N=2$ takes the form given by (C2), and then we show that (C2) holds for the next value $N+1$. For $N=2, \boldsymbol{L}_{\mathrm{QUMI}}$ just reduces to the symplectic transformation describing a $50 / 50$ beam splitter [51], i.e.,

$$
\boldsymbol{L}_{\mathrm{QUMI}}=\sqrt{\frac{1}{2}}\left(\begin{array}{cc}
\boldsymbol{I}_{2} & \boldsymbol{I}_{2} \\
-\boldsymbol{I}_{2} & \boldsymbol{I}_{2}
\end{array}\right),
$$

with $I_{2}$ being the $N \times N$ identity matrix. For $N=3$, we must realize that both the probe third and second mode interfere with transmissivity $\tau_{2}=1 / 2$, and subsequently the probe second mode interferes with the probe first mode with transmissivity $\tau_{3}=2 / 3$. Hence, $\boldsymbol{L}_{\mathrm{QUMI}}$ must result from two subsequent beam splitter operations, i.e.,

$$
\boldsymbol{L}_{\mathrm{QUMI}}=\left(\begin{array}{ccc}
\sqrt{1 / 3} \boldsymbol{I}_{2} & \sqrt{1-1 / 3} \boldsymbol{I}_{2} & \mathbf{0}_{2} \\
-\sqrt{1-1 / 3} \boldsymbol{I}_{2} & \sqrt{1 / 3} \boldsymbol{I}_{2} & \mathbf{0}_{2} \\
\mathbf{0}_{2} & \mathbf{0}_{2} & \boldsymbol{I}_{2}
\end{array}\right)\left(\begin{array}{ccc}
\boldsymbol{I}_{2} & \mathbf{0}_{2} & \mathbf{0}_{2} \\
\mathbf{0}_{2} & \sqrt{1 / 2} \boldsymbol{I}_{2} & \sqrt{1-1 / 2} \boldsymbol{I}_{2} \\
\mathbf{0}_{2} & -\sqrt{1-1 / 2} \boldsymbol{I}_{2} & \sqrt{1 / 2} \boldsymbol{I}_{2}
\end{array}\right)
$$

where $\mathbf{0}_{2}$ denotes the $N \times N$ zero matrix. After manipulation the above expression returns the result expected from (C2) for $N=3$. We can repeat this procedure for $N=4$ in similar fashion, i.e.,

$$
\begin{aligned}
\boldsymbol{L}_{\mathrm{QUMI}}= & \left(\begin{array}{cccc}
\sqrt{1 / 4} \boldsymbol{I}_{2} & \sqrt{1-1 / 4} \boldsymbol{I}_{2} & \mathbf{0}_{2} & \mathbf{0}_{2} \\
-\sqrt{1-1 / 4} \boldsymbol{I}_{2} & \sqrt{1 / 4} \boldsymbol{I}_{2} & \mathbf{0}_{2} & \mathbf{0}_{2} \\
\mathbf{0}_{2} & \mathbf{0}_{2} & \boldsymbol{I}_{2} & \mathbf{0}_{2} \\
\mathbf{0}_{2} & \mathbf{0}_{2} & \mathbf{0}_{2} & \boldsymbol{I}_{2}
\end{array}\right)\left(\begin{array}{cccc}
\boldsymbol{I}_{2} & \mathbf{0}_{2} & \mathbf{0}_{2} & \mathbf{0}_{2} \\
\mathbf{0}_{2} & \sqrt{1 / 3} \boldsymbol{I}_{2} & \sqrt{1-1 / 3} \boldsymbol{I}_{2} & \mathbf{0}_{2} \\
\mathbf{0}_{2} & -\sqrt{1-1 / 3} \boldsymbol{I}_{2} & \sqrt{1 / 3} \boldsymbol{I}_{2} & \mathbf{0}_{2} \\
\mathbf{0}_{2} & \mathbf{0}_{2} & \mathbf{0}_{2} & \boldsymbol{I}_{2}
\end{array}\right) \\
& \times\left(\begin{array}{cccc}
\boldsymbol{I}_{2} & \mathbf{0}_{2} & \mathbf{0}_{2} & \mathbf{0}_{2} \\
\mathbf{0}_{2} & \boldsymbol{I}_{2} & \mathbf{0}_{2} & \mathbf{0}_{2} \\
\mathbf{0}_{2} & \mathbf{0}_{2} & \sqrt{1 / 2} \boldsymbol{I}_{2} & \sqrt{1-1 / 2} \boldsymbol{I}_{2} \\
\mathbf{0}_{2} & \mathbf{0}_{2} & -\sqrt{1-1 / 2} \boldsymbol{I}_{2} & \sqrt{1 / 2} \boldsymbol{I}_{2}
\end{array}\right)
\end{aligned}
$$

and probe that $\boldsymbol{L}_{\mathrm{QUMI}}$ for $N=4$ coincides again with the orthogonal matrix retrieved by (C2). By repeating this procedure one may see that $\boldsymbol{L}_{\mathrm{QUMI}}$ for arbitrary size $N$ takes the form provided by the expression (C2), as we wanted to show.

By computing Eq. (32) replacing (C2) for different small values of $N$, an induction procedure for greater $N$ reveals that

$$
\boldsymbol{\Omega}_{N}\left(\varphi, s_{1}, s_{2}\right)=\left(\begin{array}{cccc}
d_{1} & c_{1} & c_{2} & \frac{c_{3}}{s_{1} s_{2}} \\
c_{1} & d_{2} & c_{3} & -\frac{c_{2}}{s_{1} s_{2}} \\
c_{2} & c_{3} & d_{3} & 0 \\
\frac{c_{3}}{s_{1} s_{2}} & -\frac{c_{2}}{s_{1} s_{2}} & 0 & d_{4}
\end{array}\right),
$$

the diagonal entries of which are determined by

$$
\begin{gathered}
d_{1}=\frac{1}{s_{1} s_{2} N}\left(s_{1} s_{2} a_{N}\left(s_{2}, s_{1}\right) \cos ^{2} \varphi+a_{N}\left(s_{1}, s_{2}\right) \sin ^{2} \varphi\right) \\
d_{2}=\frac{1}{s_{1} s_{2} N}\left(a_{N}\left(s_{1}, s_{2}\right) \cos ^{2} \varphi+s_{1} s_{2} a_{N}\left(s_{2}, s_{1}\right) \sin ^{2} \varphi\right), \\
d_{3}=\frac{a_{N}\left(s_{1}, s_{2}\right)}{N}, \\
d_{4}=\frac{a_{N}\left(s_{2}, s_{1}\right)}{s_{1} s_{2} N}
\end{gathered}
$$

whereas the nondiagonal elements are given by

$$
\begin{gathered}
c_{1}=\frac{a_{N}\left(s_{1}, s_{2}\right)-s_{1} s_{2} a_{N}\left(s_{2}, s_{1}\right)}{2 s_{1} s_{2} N} \sin (2 \varphi), \\
c_{2}=\frac{\left(s_{2}-s_{1}\right) \sqrt{N-1}}{N} \cos \varphi \\
c_{3}=\frac{\left(s_{1}-s_{2}\right) \sqrt{N-1}}{N} \sin \varphi .
\end{gathered}
$$


On the other side, by replacing the generic form (C3) in (33) and using results borrowed from matrix analysis to compute the Moore-Penrose pseudoinverse [64], one obtains the auxiliary matrix (33) with

$$
\boldsymbol{A}^{(x)}=\left(\begin{array}{cccc}
\frac{d_{3}}{d_{1} d_{3}-c_{2}^{2}} & 0 & -\frac{c_{2}}{d_{1} d_{3}-c_{2}^{2}} & 0 \\
0 & 0 & 0 & 0 \\
-\frac{c_{2}}{d_{1} d_{3}-c_{2}^{2}} & 0 & \frac{d_{1}}{d_{1} d_{3}-c_{2}^{2}} & 0 \\
0 & 0 & 0 & 0
\end{array}\right),
$$

whereas for the momentum quadrature measurement

$$
\boldsymbol{A}^{(p)}=\left(\begin{array}{cccc}
0 & 0 & 0 & 0 \\
0 & \frac{d_{4} s_{1}^{2} s_{2}^{2}}{d_{2} d_{4} s_{1}^{2} s_{2}^{2}-c_{2}^{2}} & 0 & \frac{c_{2} s_{1} s_{2}}{d_{2} d_{4} s_{1}^{2} s_{2}^{2}-c_{2}^{2}} \\
0 & 0 & 0 & 0 \\
0 & \frac{c_{2} s_{1} s_{2}}{d_{2} d_{4} s_{1}^{2} s_{2}^{2}-c_{2}^{2}} & 0 & \frac{d_{2} s_{1}^{2} s_{2}^{2}}{d_{2} d_{4} s_{1}^{2} s_{2}^{2}-c_{2}^{2}}
\end{array}\right),
$$

which reduces to the expected results $\boldsymbol{A}^{(x)}=\operatorname{diag}(1,0,1,0)$ or $\boldsymbol{A}^{(p)}=\operatorname{diag}(0,1,0,1)$ when the initial squeezing vanishes [51] (i.e., $s_{1}=s_{2}=1$ ).

Moreover, by substituting (C3) in (28), one obtains the expression (34) for the FI for position quadrature measurement after a long tedious calculation where we have introduced

$$
\begin{gathered}
\left(\boldsymbol{W}_{N}^{(x)}\right)_{11}=-\frac{i N s_{1} s_{2}}{2 N s_{1} s_{2} \cot (\varphi)+2 i\left((N-1) s_{1}+s_{2}\right)}+\frac{i N s_{1} s_{2} \sin (\varphi)}{2 N s_{1} s_{2} \cos (\varphi)-2 i \sin (\varphi)\left((N-1) s_{1}+s_{2}\right)}+\frac{s_{1}-s_{2}}{N}+s_{2} \\
\left(\boldsymbol{W}_{N}^{(x)}\right)_{22}=\frac{\left((N-1) s_{1}+s_{2}\right)^{3}}{N^{3} s_{1}^{3} s_{2}^{3} \cot ^{2}(\varphi)+N s_{1} s_{2}\left((N-1) s_{1}+s_{2}\right)^{2}} \\
\left(\boldsymbol{W}_{N}^{(x)}\right)_{12}=\left(\boldsymbol{W}_{N}^{(x)}\right)_{21}=\frac{N s_{1} s_{2} \sin (2 \varphi)\left((N-1) s_{1}+s_{2}\right)}{2 N^{2} s_{1}^{2} s_{2}^{2} \cos ^{2}(\varphi)+2 \sin ^{2}(\varphi)\left((N-1) s_{1}+s_{2}\right)^{2}}
\end{gathered}
$$

or for the momentum quadrature measurement

$$
\begin{gathered}
\left(\boldsymbol{W}_{N}^{(p)}\right)_{11}=\frac{\left((N-1) s_{2}+s_{1}\right)^{3}}{N^{3} \cot ^{2}(\varphi)+N\left((N-1) s_{2}+s_{1}\right)^{2}}, \\
\left(\boldsymbol{W}_{N}^{(p)}\right)_{22}=-\frac{\frac{1}{s_{1}}-\frac{1}{s_{2}}}{N}+\frac{1}{N_{2} \cot ^{2}(\varphi)+\left((N-1) s_{2}+s_{1}\right)} \\
\left(\boldsymbol{W}_{N}^{(p)}\right)_{12}=\left(\boldsymbol{W}_{N}^{(p)}\right)_{21}=-\frac{N \sin (2 \varphi)\left((N-1) s_{2}+s_{1}\right)}{2\left(N^{2} \cos ^{2}(\varphi)+\sin ^{2}(\varphi)\left((N-1) s_{2}+s_{1}\right)^{2}\right)},
\end{gathered}
$$

as well as the auxiliary functions determining the influence of the second-moment resources:

$$
\begin{aligned}
f_{N}^{(x)}\left(\sin ^{2} \varphi, s_{1}, s_{2}\right)= & \left((N-1) s_{1} s_{2}^{5}\left(N^{2} s_{1}^{2}+1\right)+2 s_{2}^{4}\left(s_{1}^{2}\left(N^{2}((N-1) N+1) s_{1}^{2}-N(N+3)+2\right)+1\right)\right. \\
& -2(N-1)^{2} s_{1}^{2} s_{2}^{2}\left(\left(2 N^{2}+N-2\right) s_{1}^{2}-6\right)+2(N-1)^{4} s_{1}^{4}+(N-1) s_{1} s_{2}^{3}\left(s_{1}^{2}\left(N\left(N\left(s_{1}^{2}-7\right)-6\right)+6\right)+8\right) \\
& +\cos (2 \varphi)\left(N s_{1}\left(s_{2}-1\right)+s_{1}-s_{2}\right)\left(2 s_{2}^{2}\left(((N-1) N+1) s_{1}^{2}-1\right)+(N-1) s_{1}\left(s_{1}^{2}-4\right) s_{2}\right. \\
& \left.\left.-2(N-1)^{2} s_{1}^{2}+(N-1) s_{1} s_{2}^{3}\right)\left(s_{1}\left(N s_{2}+N-1\right)+s_{2}\right)+(N-1)^{3} s_{1}^{3}\left(s_{1}^{2}+8\right) s_{2}\right) \frac{\sin ^{2} \varphi}{2 y_{x}^{2}(\varphi)}
\end{aligned}
$$

or

$$
\begin{aligned}
f_{N}^{(p)}\left(\sin ^{2} \varphi, s_{1}, s_{2}\right)= & \left(2 N^{4} s_{1} s_{2}\left(s_{2}^{2}-1\right)^{2}+N^{3}\left(s_{1}-s_{2}\right)\left(8 s_{1} s_{2}^{4}-7 s_{1} s_{2}^{2}+s_{1}-s_{2}^{3}-s_{2}\right)\right. \\
& +\cos (2 \varphi)\left(N\left(s_{2}-1\right)+s_{1}-s_{2}\right)\left((N-1) s_{2}+N+s_{1}\right)\left(2 s_{1} s_{2}\left(-N^{2}+N+s_{1}^{2}-1\right)\right. \\
& \left.+(N-1)\left(4 s_{1}^{2}-1\right) s_{2}^{2}+(1-N) s_{1}^{2}+2(N-1)^{2} s_{1} s_{2}^{3}\right)+N^{2}\left(s_{1}-s_{2}\right)^{2}\left(12 s_{1} s_{2}^{3}-2 s_{1} s_{2}-3 s_{2}^{2}-1\right) \\
& \left.+N\left(s_{1}-s_{2}\right)^{3}\left(8 s_{1} s_{2}^{2}+s_{1}-3 s_{2}\right)+\left(s_{1}-s_{2}\right)^{4}\left(2 s_{1} s_{2}-1\right)\right) \frac{\sin ^{2} \varphi}{2 s_{1} s_{2} y_{p}^{2}(\varphi)}
\end{aligned}
$$

with $y_{x}(\varphi)=\left(N s_{1} s_{2}\right)^{2} \cos ^{2} \varphi+a_{N}^{2}\left(s_{1}, s_{2}\right) \sin ^{2} \varphi$ and $y_{p}(\varphi)=\left(N^{2} \cos ^{2} \varphi+a_{N}^{2}\left(s_{2}, s_{1}\right) \sin ^{2} \varphi\right.$. 
When addressing the optimal working point, as stated in the main text [by demanding the second and third terms in the right-hand side of Eq. (34) cancel] we find out the second-order polynomial (38) with real coefficients given by

$$
\begin{aligned}
& \alpha_{N}^{(x)}\left(s_{1}, s_{2}\right)=-2 N^{2} s_{1}^{2} s_{2}^{2}\left((N-1) s_{1}+s_{2}\right)^{2}\left(2 N^{2}-\left(\frac{N-1}{s_{2}}+\frac{1}{s_{1}}\right)^{2}-\left((N-1) s_{2}+s_{1}\right)^{2}\right) \\
&-2 N^{2}\left(N s_{1}\left(s_{2}-1\right)+s_{1}-s_{2}\right)\left(2 s_{2}^{2}\left(((N-1) N+1) s_{1}^{2}-1\right)+(N-1) s_{1}\left(s_{1}^{2}-4\right) s_{2}-2(N-1)^{2} s_{1}^{2}\right. \\
&\left.+(N-1) s_{1} s_{2}^{3}\right)\left(s_{1}\left(N s_{2}+N-1\right)+s_{2}\right)+\left((N-1) s_{1}+s_{2}\right)^{4}\left(2 N^{2}-\left(\frac{N-1}{s_{2}}+\frac{1}{s_{1}}\right)^{2}-\left((N-1) s_{2}+s_{1}\right)^{2}\right) \\
&+N^{4} s_{1}^{4} s_{2}^{4}\left(2 N^{2}-\left(\frac{N-1}{s_{2}}+\frac{1}{s_{1}}\right)^{2}-\left((N-1) s_{2}+s_{1}\right)^{2}\right) \\
& \beta_{N}^{(x)}\left(s_{1}, s_{2}\right)= 2 N^{2}\left((N-1)^{2} s_{1}^{2} s_{2}^{6}\left(N^{2} s_{1}^{2}-1\right)-(N-1) s_{1} s_{2}^{3}\left(2 N^{2} s_{1}^{2}+(N-2)^{2} s_{1}^{4}-4\right)\right. \\
&-(N-1)^{2} s_{1}^{2} s_{2}^{2}\left(N^{2} s_{1}^{2}+s_{1}^{4}-6\right)+s_{2}^{4}\left(N^{2} s_{1}^{6}-N^{2} s_{1}^{2}-(N(N((N-2) N+8)-12)+6) s_{1}^{4}+1\right) \\
&\left.+(N-1)^{4} s_{1}^{4}+4(N-1)^{3} s_{1}^{3} s_{2}+(N-1) s_{1}^{3} s_{2}^{5}\left(N\left(N\left(2 s_{1}^{2}-1\right)+4\right)-4\right)\right) \\
& \delta_{N}^{(x)}\left(s_{1}, s_{2}\right)=N^{4} s_{1}^{4} s_{2}^{4}\left(2 N^{2}-\left(\frac{N-1}{s_{2}}+\frac{1}{s_{1}}\right)^{2}-\left((N-1) s_{2}+s_{1}\right)^{2}\right)
\end{aligned}
$$

\section{Explicit expressions from Sec. III B}

Now we turn the attention to the polychromatic scenario described in Sec. III B. As stated in the discussion about the Fisher information, after some manipulation one can show that for the choices $\epsilon= \pm 1$ the expression (45) boils down to

$$
F_{\mathrm{pol}}^{(x)}(\varphi, \epsilon)=\mathcal{F}_{\mathrm{pol}}(\epsilon)-\frac{2\left(\operatorname{sign}(\epsilon) \sinh ^{2}\left(2 s^{\prime}\right)+\cos (4 \varphi) \sinh \left(4 s^{\prime}\right)\right)^{2}}{\left(\cosh \left(2 s^{\prime}\right)+\operatorname{sign}(\epsilon) \cos (4 \varphi) \sinh \left(2 s^{\prime}\right)\right)^{2}},
$$

for a measurement quadrature in position, or

$$
F_{\mathrm{pol}}^{(p)}(\varphi, \epsilon)=\mathcal{F}_{\mathrm{pol}}(\epsilon)-\frac{2\left(\operatorname{sign}(\epsilon) \sinh ^{2}\left(2 s^{\prime}\right)-\cos (4 \varphi) \sinh \left(4 s^{\prime}\right)\right)^{2}}{\left(\cosh \left(2 s^{\prime}\right)-\operatorname{sign}(\epsilon) \cos (4 \varphi) \sinh \left(2 s^{\prime}\right)\right)^{2}},
$$

for a measurement quadrature in momentum. By paying attention to Eqs. (C24) and (C25), it is clear that the optimal operating point is obtained by demanding the numerator of the second term in the right-hand side cancels. Upon doing this, one arrives to the relation determining the optimal angle for the choice $\epsilon= \pm 1$. For the most general case of modulation frequency (i.e., $\epsilon \neq \pm 1$ ), one obtains the following subsidiary condition from a perturbative analysis:

$$
\left(3+\cosh \left(4 s^{\prime}\right)-2 \cos (4 \phi) \sinh ^{2}\left(2 s^{\prime}\right)\right) f_{0}^{(x)}\left(\cos (4 \phi), s^{\prime}\right)+\epsilon f_{1}^{(x)}\left(\phi, s^{\prime}\right) \approx 0,
$$

where we have introduced the auxiliary functions

$$
\begin{aligned}
f_{0}^{(x)}\left(\cos (4 \varphi), s^{\prime}\right)= & \sinh ^{2}(2 r)\left(-2 \sinh ^{2}(2 r) \cos (4 \varphi)+\cosh (4 r)+3\right)\left(2 \sinh ^{2}(4 r)\left(2 \cos ^{2}(4 \varphi)-1\right)\right. \\
& -4(\cosh (8 r)-9) \cos (4 \varphi)+3 \cosh (8 r)+29), \\
f_{1}^{(x)}\left(\varphi, s^{\prime}\right)= & 16 \sinh ^{3}(2 r)(2 \varphi \sinh (2 r) \sinh (4 r) \sin (10 \varphi)+\cosh (6 r)(-14 \varphi \sin (2 \varphi)+3 \varphi \sin (6 \varphi)-3 \cos (6 \varphi)) \\
+ & \cosh (2 r)\left(4(\cosh (4 r)+3) \cos (2 \varphi)+16 \sinh ^{2}(r) \cosh ^{2}(r) \cos (10 \varphi)-2 \varphi \sin (2 \varphi)+45 \varphi \sin (6 \varphi)\right. \\
& -13 \cos (6 \varphi))) .
\end{aligned}
$$

Clearly, from Eq. (C26) follows that in the particular case $\epsilon=0$ the optimal operating point $\varphi_{\mathrm{opt}}^{(x)}$ is figured out from solving the second-order polynomial $f_{0}^{(x)}\left(y, s^{\prime}\right)=0$ with argument understood as $y=\cos (4 \varphi)$. Doing this, one directly obtains

$$
y=\frac{1}{2}(\cosh (8 r) \pm 4 \sqrt{6-2 \cosh (8 r)}-9) \operatorname{csch}^{2}(4 r),
$$

which is greater than the unit except for the choice of coherent resources $s^{\prime}=0$ when one of the roots becomes $x \rightarrow-1$, retrieving in turn the same result $\varphi_{\mathrm{opt}}^{(x)}=-\pi / 4$ as previously obtained in Sec. III A, as expected.

\section{Explicit expressions from Sec. III C}

In this Appendix, we briefly illustrate the derivation of Eqs. (47), (48), and (49) appearing in Sec. III C. First, the nonunit efficiency of a single-mode homodyne measurement mainly resides in the use of photon detectors suffering from 
a limited resolution $\eta_{\text {eff }} \in[0,1]$, which results in a vacuum noise contribution proportional to $\sqrt{1-\eta_{\mathrm{eff}}}$ in the measurement outcomes, i.e.,

$$
[\lambda]=\frac{1}{2}\left(\sqrt{\frac{1+r}{2}} q^{f}, \sqrt{\frac{1+r}{2 r}} p^{f}\right)+\sqrt{1-\eta_{\mathrm{eff}}}\left(q^{\mathrm{vac}}, p^{\mathrm{vac}}\right) .
$$

Without loss of generality, this source of noise may be well approximated by the combination of an ideal Gaussian detector (described by the CV matrix $\Sigma$ ) preceding by a beam splitter with transmission coefficient identical to the photon-detector resolution factor, where the probe mode would fictitiously interfere with an input vacuum beam representing $\left(\hat{q}^{\mathrm{vac}}, \hat{p}^{\mathrm{vac}}\right)$. In our framework, this corresponds to take the CV matrix determining the nonideal Gaussian measurement scheme as

$$
\boldsymbol{\Sigma}=\eta_{\mathrm{eff}} \boldsymbol{\Sigma}+\left(1-\eta_{\mathrm{eff}}\right) \boldsymbol{I}_{N}
$$

which returns the lossless homodyne detection scenario for $\eta_{\text {eff }}=1$. On the other side, decoherence effects of the probe $N$-mode system taking place during the light field propagation through the interferometer can be formulated in terms of the interaction with an environment modeled by a continuum of oscillators [87]. When the system-environment interaction is essentially linear, the time evolution of our probe $N$-mode system is governed by the Fokker-Plank (or diffusion) equation expressed in the interaction picture [86]:

$$
\frac{\partial W(\boldsymbol{R}, t)}{\partial t}=\left(\left(\frac{\partial}{\partial \boldsymbol{R}}\right)^{T} \boldsymbol{\Gamma} \boldsymbol{R}+\left(\frac{\partial}{\partial \boldsymbol{R}}\right)^{T} \boldsymbol{D}\left(\frac{\partial}{\partial \boldsymbol{R}}\right)\right) W(\boldsymbol{R}, t),
$$

with $\left(\frac{\partial}{\partial \boldsymbol{R}}\right)^{T}=\bigoplus_{i=1}^{N}\left(\frac{\partial}{\partial q_{i}}, \frac{\partial}{\partial p_{i}}\right) ; \boldsymbol{\Gamma}$ and $\boldsymbol{D}$ are $2 N \times 2 N$ real, symmetric matrices that essentially encrypt the photon losses and thermal noise effects, respectively. In the interesting dissipative scenario the above matrices take the following simple form:

$$
\begin{gathered}
\boldsymbol{\Gamma}=\frac{\gamma}{2} \bigoplus_{i=1}^{N} \boldsymbol{I}_{1}, \\
\boldsymbol{D}=\frac{\gamma\left(1+2 n_{\mathrm{th}}\right)}{4} \bigoplus_{i=1}^{N} \boldsymbol{I}_{1},
\end{gathered}
$$

where $\gamma$ is the usual dissipative coefficient. Equation (C32) is a linear Fokker-Plank equation that can be solved by using the Green's-function method [86]. Furthermore, thanks to the diagonal form of the above dissipative and noise matrices, the decoherence evolution commutes with the phase shift rotation [22,38,81], and we obtain the results (47) and (48). Substituting these in Eq. (28), and following a similar procedure as to compute the expression (23), we obtain

$$
\begin{aligned}
F_{\text {deco }}\left(\varphi, \eta_{\text {loss }}, \eta_{\text {eff }}, n_{\text {th }}\right)= & F\left(\varphi, \eta_{\text {loss }}, \eta_{\text {eff }}\right)-\eta_{\text {loss }}\left\langle\boldsymbol{R}^{T}\right\rangle \boldsymbol{L}^{T} \boldsymbol{P}_{\varphi}^{T} \\
& \times \boldsymbol{\Sigma}_{\text {deco }}^{-1} \boldsymbol{P}_{\varphi} \boldsymbol{L}\langle\boldsymbol{R}\rangle \\
& +\frac{1}{2} \operatorname{Tr}\left(\partial_{\varphi} \boldsymbol{\Sigma}_{\text {deco }}^{-1} \partial_{\varphi} \boldsymbol{\sigma}_{\text {deco }}\right),
\end{aligned}
$$

with

$$
\begin{aligned}
\boldsymbol{\Sigma}_{\mathrm{deco}}\left(\eta_{\text {loss }}, \eta_{\mathrm{eff}}, n_{\mathrm{th}}\right)= & \boldsymbol{\sigma}_{\mathrm{deco}}\left(\left(1-\eta_{\mathrm{eff}}+\left(1-\eta_{\text {loss }}\right)\right.\right. \\
& \left.\left.\times\left(1+n_{\mathrm{th}}\right)\right)^{-1} \boldsymbol{I}_{N}+\boldsymbol{\sigma}_{\mathrm{deco}}^{-1}\right) \boldsymbol{\sigma}_{\mathrm{deco}},
\end{aligned}
$$

where $F\left(\varphi, \eta_{\text {loss }}, \eta_{\text {eff }}\right)$ comprises the Fisher information obtained from the noiseless expression (28) after substituting $\boldsymbol{S}(\varphi) \rightarrow \sqrt{\eta_{\text {loss }}} \boldsymbol{S}(\varphi)$, and $\boldsymbol{\Sigma} \rightarrow \eta_{\mathrm{eff}} \boldsymbol{\Sigma}$. It is worthwhile to realize that the second contribution in the right-hand side of (C35) will be always negative for any $\varphi \in \mathbb{R}$ and $\langle\boldsymbol{R}\rangle \in \mathbb{R}^{2 N}$, since the CV matrix $\boldsymbol{\Sigma}_{\text {deco }}^{-1}$ is positive-semidefinite by construction, and further it asymptotically cancels in the limit of an ideal phase-estimation scenario (i.e., $F_{\text {deco }} \rightarrow F$ when $\eta_{\text {eff }}, \eta_{\text {loss }} \rightarrow 1$ ), as expected. Notice that the corresponding QFI is formally obtained from Eq. (17) (with $n_{t}=0$ ) by replacing $\left\langle\boldsymbol{R}_{1}^{\prime}\right\rangle \rightarrow \sqrt{\eta_{\text {loss }}} \boldsymbol{P}_{0} \boldsymbol{L}\langle\boldsymbol{R}\rangle$, and $\boldsymbol{V}_{1}^{\prime} \rightarrow \boldsymbol{P}_{0}\left(\eta_{\text {loss }} \boldsymbol{L} \boldsymbol{V} \boldsymbol{L}^{T}+\right.$ $\left.\left(1-\eta_{\text {loss }}\right)\left(1+n_{\text {th }}\right) \boldsymbol{I}_{N}\right) \boldsymbol{P}_{0}^{T}$.

In particular, in the dissipative scenario $\eta_{\text {eff }}=\eta_{\text {loss }}=\eta$, we find the FI for the previously studied coherent resources and homodyne detection, i.e.,

$$
\begin{aligned}
F_{\mathrm{deco}}^{(x / p)}\left(\varphi, \eta, n_{t h}\right)= & 2 \bar{n}_{c} N(1 \mp \sin (2 \varphi)) \\
& \times\left(\eta^{2}+\frac{\left(2+n_{\mathrm{th}}\right)(1-\eta)}{\eta+(1-\eta) n_{\mathrm{th}}-2}\right) \\
= & 2 \tilde{\eta}^{2} \bar{n}_{c} N(1 \mp \sin (2 \varphi)) .
\end{aligned}
$$

By comparing with the ideal result (27) for $\mathcal{F}$, it is clear from the above equation (C37) that the optimal working point defined in Sec. III C is obtained from demanding $\sin \left(2 \varphi^{(x / p)}\right)=$ $\mp\left[2(\eta / \tilde{\eta})^{2}-1\right]$, which returns a result that substantially differs from the ideal case (i.e., $\varphi=\mp \pi / 4$ ).
[1] A. Serafini, Quantum Continuous Variables: A Primer of Theoretical Methods (CRC, Boca Raton, FL, 2017).

[2] Z. Jiang, Phys. Rev. A 89, 032128 (2014).

[3] D. Šafránek, A. R. Lee, and I. Fuentes, New J. Phys. 17, 073016 (2015).

[4] Y. Gao and H. Lee, Eur. Phys. J. D 68, 347 (2014).

[5] A. Monras, arXiv:1303.3682v1.

[6] V. Giovannetti, S. Lloyd, and L. Maccone, Science 306, 1330 (2004).

[7] V. Giovannetti, S. Lloyd, and L. Maccone, Nat. Photonics 5, 222 (2011).
[8] M. G. A. Paris, Int. J. Quant. Inf. 7, 125 (2009).

[9] G. Tóth and I. Apellaniz, J. Phys. A: Math Theor. 47, 424006 (2014).

[10] J. P. Dowling, Contemporary Physics 49, 125 (2008).

[11] D. Braun, G. Adesso, F. Benatti, R. Floreanini, U. Marzolino, M. W. Mitchell, and S. Pirandola, Rev. Mod. Phys. 90, 035006 (2018).

[12] S. Pirandola, B. R. Bardhan, T. Gehring, C. Weedbrook, and S. Lloyd, Nat. Photonics 12, 724 (2018).

[13] H. Lee, P. Kok, and J. P. Dowling, J. Mod. Opt. 49, 2325 (2002). 
[14] R. Demkowicz-Dobrzański, M. Jarzyna, and J. Kołodyński, Prog. Opt. 60, 345 (2015).

[15] J. S. Sidhu and P. Kok, AVS Quantum Sci. 2, 014701 (2014).

[16] S. Ataman, Phys. Rev. A 100, 063821 (2019).

[17] S. Ataman, Phys. Rev. A 102, 013704 (2020).

[18] M. Gessner, A. Smerzi, and L. Pezzè, Nat. Commun. 11, 3817 (2020).

[19] S. L. Braunstein and C. M. Caves, Phys. Rev. Lett. 72, 3439 (1994).

[20] D. Šafránek, J. Phys. A: Math. Theor. 52, 035304 (2019).

[21] B. T. Gard, C. You, D. K. Mishra, R. Singh, H. Lee, T. R. Corbitt, and J. P. Dowling, EPJ Quantum Technol. 4, 4 (2017).

[22] C. Oh, S.-y. Lee, H. Nha, and H. Jeong, Phys. Rev. A 96, 062304 (2017).

[23] O. Steuernagel and S. Scheel, J. Opt. B: Quantum and Semiclass. Opt. 6, 566 (2004).

[24] R. S. Bondurant and J. H. Shapiro, Phys. Rev. D 30, 2548 (1984).

[25] D. Li, C. H. Yuan, Z. Y. Ou, and W. Zhang, New J. Phys. 16, 073020 (2014).

[26] O. Pinel, J. Fade, D. Braun, P. Jian, N. Treps, and C. Fabre, Phys. Rev. A 85, 010101(R) (2012).

[27] C. N. Gagatsos, D. Branford, and A. Datta, Phys. Rev. A 94, 042342 (2016).

[28] R. Nichols, P. Liuzzo-Scorpo, P. A. Knott, and G. Adesso, Phys. Rev. A 98, 012114 (2018).

[29] T. Matsubara, P. Facchi, V. Giovannetti, and K. Yuasa, New J. Phys. 21, 033014 (2019).

[30] C. Oh, C. Lee, L. Banchi, S.-y. Lee, C. Rockstuhl, and H. Jeong, Phys. Rev. A 100, 012323 (2019).

[31] D. Šafránek and I. Fuentes, Phys. Rev. A 94, 062313 (2016).

[32] C. Oh, C. Lee, S. H. Lie, and H. Jeong, Phys. Rev. Research 2, 023030 (2020).

[33] H. Yonezawa, D. Nakane, T. A. Wheatley, K. Iwasawa, S. Takeda, H. Arao, K. Ohki, K. Tsumura, D. W. Berry, T. C. Ralph, H. M. Wiseman, E. H. Huntington, and A. Furusawa, Science 337, 1514 (2012).

[34] S. Slussarenko, Nat. Photonics 11, 700 (2017).

[35] J. A. et al., Nat. Photonics 7, 613 (2013).

[36] M. Takeoka, K. P. Seshadreesan, C. You, S. Izumi, and J. P. Dowling, Phys. Rev. A 96, 052118 (2017).

[37] M. Jarzyna and R. Demkowicz-Dobrzański, Phys. Rev. A 85, 011801(R) (2012).

[38] C. Oh, C. Lee, C. Rockstuhl, H. Jeong, J. Kim, H. Nha, and S.-Y. Lee, npj Quantum Inf. 5, 10 (2018).

[39] Z. Chaboyer, T. Meany, L. G. Helt, M. J. Withford, and M. J. Steel, Sci. Rep. 5, 10 (2015).

[40] G. Gramegna, D. Triggiani, P. Facchi, F. A. Narducci, and V. Tamma, arXiv:2003.12550.

[41] G. Gramegna, D. Triggiani, P. Facchi, F. A. Narducci, and V. Tamma, Phys. Rev. Research 3, 013152 (2021).

[42] E. Polino, M. Riva, M. Valeri, R. Silvestri, G. Corrielli, A. Crespi, N. Spagnolo, R. Osellame, and F. Sciarrino, Optica 6, 288 (2019).

[43] S. Paesani, A. A. Gentile, R. Santagati, J. Wang, N. Wiebe, D. P. Tew, J. L. O’Brien, and M. G. Thompson, Phys. Rev. Lett. 118, 100503 (2017).

[44] O. Pinel, P. Jian, N. Treps, C. Fabre, and D. Braun, Phys. Rev. A 88, 040102(R) (2013).
[45] C. You, S. Adhikari, Y. Chi, M. L. Laborde, C. T. Matyas, C. Zhang, Z. Su, T. Byrnes, C. Lu, J. P. Dowling, and J. P. Olson, J. Opt. 19, 124002 (2017).

[46] J. P. Olson, K. R. Motes, P. M. Birchall, N. M. Studer, M. LaBorde, T. Moulder, P. P. Rohde, and J. P. Dowling, Phys. Rev. A 96, 013810 (2017).

[47] K. R. Motes, J. P. Olson, E. J. Rabeaux, J. P. Dowling, S. J. Olson, and P. P. Rohde, Phys. Rev. Lett. 114, 170802 (2015).

[48] Z.-e. Su, Y. Li, P. P. Rohde, H.-1. Huang, X.-1. Wang, L. Li, N.-1. Liu, J. P. Dowling, C.-y. Lu, and J.-w. Pan, Phys. Rev. Lett. 119, 080502 (2017).

[49] A. Ferraro, S. Olivares, and M. Paris, Gaussian States in Continuous Variable Quantum Information (Bibliopolis, Napoli, 2005).

[50] S. L. Braunstein and P. van Loock, Rev. Mod. Phys. 77, 513 (2005).

[51] C. Weedbrook, S. Pirandola, R. García-Patrón, N. J. Cerf, T. C. Ralph, J. H. Shapiro, and S. Lloyd, Rev. Mod. Phys. 84, 621 (2012).

[52] S. Boixo, S. T. Flammia, C. M. Caves, and J. M. Geremia, Phys. Rev. Lett. 98, 090401 (2007).

[53] J. Mathieu Elias Fraïsse and D. Braun, Phys. Rev. A 95, 062342 (2017).

[54] A. Monras, Phys. Rev. A 73, 033821 (2006).

[55] M. Aspachs, J. Calsamiglia, R. Muñoz-Tapia, and E. Bagan, Phys. Rev. A 79, 033834 (2009).

[56] C. Sparaciari, S. Olivares, and M. G. A. Paris, Phys. Rev. A 93, 023810 (2016).

[57] S. Olivares, Eur. Phys. J. Special Topics 203, 3 (2012).

[58] G. Giedke and J. Ignacio Cirac, Phys. Rev. A 66, 032316 (2002).

[59] M. G. Genoni, S. Mancini, and A. Serafini, Russ. J. Math. Phys. 21, 329 (2014).

[60] M. S. Kim and B. C. Sanders, Phys. Rev. A 53, 3694 (1996).

[61] L. Pezzé and A. Smerzi, Phys. Rev. Lett. 100, 073601 (2008).

[62] S. L. Braunstein, C. M. Caves, and G. J. Milburn, Ann. Phys. (NY) 173, 135 (1996).

[63] L. Pezzé, M. A. Ciampini, N. Spagnolo, P. C. Humphreys, A. Datta, I. A. Walmsley, M. Barbieri, F. Sciarrino, and A. Smerzi, Phys. Rev. Lett. 119, 130504 (2017).

[64] K. Petersen and M. Pedersen, The Matrix Cookbook (Technical University of Denmark, 2012).

[65] D. Bernstein, Matrix Mathematics: Theory, Facts, and Formulas with Application to Linear Systems Theory (Princeton University, Princeton, NJ, 2002).

[66] J. Eisert, S. Scheel, and M. B. Plenio, Phys. Rev. Lett. 89, 137903 (2002).

[67] Physically, the tensorial product of identical Gaussian states (up to an arbitrary displacement) with a diagonal CV matrix remains invariant under a beam splitter transformation (which induces no phase shift between transmitted and reflected modes) for any transmission coefficient $[79,89]$.

[68] S. Olivares and M. G. Paris, J. Phys. B: At. Mol. Opt. Phys. 42, 055506 (2009).

[69] A. DePasquale, P. Facchi, G. Florio, V. Giovannetti, K. Matsuoka, and K. Yuasa, Phys. Rev. A 92, 042115 (2015).

[70] R. Gaiba and M. G. Paris, Phys. Lett. A 373, 934 (2009).

[71] M. D. Lang and C. M. Caves, Phys. Rev. A 90, 025802 (2014).

[72] C. Sparaciari, S. Olivares, and M. G. A. Paris, J. Opt. Soc. Am. B 32, 001354 (2015). 
[73] C. M. Caves, Phys. Rev. D 23, 1693 (1981).

[74] J.-D. Zhang, C.-F. Jin, Z.-J. Zhang, L.-Z. Cen, J.-Y. Hu, and Y. Zhao, Opt. Express 26, 33080 (2018).

[75] B. Yurke, S. L. McCall, and J. R. Klauder, Phys. Rev. A 33, 4033 (1986).

[76] P. M. Anisimov, G. M. Raterman, A. Chiruvelli, W. N. Plick, S. D. Huver, H. Lee, and J. P. Dowling, Phys. Rev. Lett. 104, 103602 (2010).

[77] W. N. Plick, P. M. Anisimov, J. P. Dowling, H. Lee, and G. S. Agarwal, New J. Phys. 12, 113025 (2010).

[78] R. Birrittella, A. Gura, and C. C. Gerry, Phys. Rev. A 91, 053801 (2015).

[79] M. S. Kim, W. Son, V. Bužek, and P. L. Knight, Phys. Rev. A 65, 032323 (2002).

[80] C. You, S. Adhikari, X. Ma, M. Sasaki, M. Takeoka, and J. P. Dowling, Phys. Rev. A 99, 042122 (2019).
[81] M. Jarzyna and M. Zwierz, Phys. Rev. A 95, 012109 (2017).

[82] Y. Gao, Phys. Rev. A 94, 023834 (2016).

[83] C. N. Gagatsos, B. A. Bash, S. Guha, and A. Datta, Phys. Rev. A 96, 062306 (2017).

[84] B. M. Escher, R. L. De Matos Filho, and L. Davidovich, Nat. Phys. 7, 406 (2011).

[85] J. Kołodynski and R. Demkowicz-Dobrzanski, New J. Phys 15, 073043 (2013).

[86] A. A. Valido, F. Levi, and F. Mintert, Phys. Rev. A 90, 052321 (2014).

[87] A. Serafini, M. G. Paris, F. Illuminati, and S. De Siena, J. Opt. B: Quantum Semiclass. Opt. 7, R19 (2005).

[88] N. C. Harris, G. R. Steinbrecher, J. Mower, Y. Lahini, M. Prabhu, T. Baehr-Jones, M. Hochberg, S. Lloyd, and D. Englund, Nat. Photonics 11, 447 (2017).

[89] S. C. Springer, J. Lee, M. Bellini, and M. S. Kim, Phys. Rev. A 79, 062303 (2009). 